\title{
Noncovalent Interactions in Organic Electronic Materials
}

\author{
Mahesh Kumar Ravva, ${ }^{1}$ Chad Risko, ${ }^{2}$ and Jean-Luc Brédas ${ }^{1}$ \\ ${ }^{1}$ Laboratory for Computational and Theoretical Chemistry of Advanced Materials \\ Division of Physical Science and Engineering \\ King Abdullah University of Science and Technology \\ Thuwal 23599-6900, Kingdom of Saudi Arabia \\ ${ }^{2}$ Department of Chemistry \\ \& Center for Applied Energy Research \\ University of Kentucky \\ Lexington, Kentucky 40506-0055
}

\begin{abstract}
In this Chapter, we provide an overview of how noncovalent interactions, determined by the chemical structure of $\pi$-conjugated molecules and polymers, govern essential aspects of the electronic, optical, and mechanical characteristics of organic semiconductors. We begin by describing general aspects of materials design, including the wide variety of chemistries exploited to control the electronic and optical properties of these materials. We then discuss explicit examples of how the study of noncovalent interactions can provide deeper chemical insights that can improve the design of new generations of organic electronic materials.
\end{abstract}




\section{Introduction}

Organic semiconductors, electronically and optically active materials derived from $\pi$-conjugated molecules and polymers, have attracted major academic and industrial attention over the past three decades. The potential for large-scale and low-cost device fabrication, distinctive device form factors that arise from the inherent mechanical flexibility of thin-film 'plastic' materials, and the ability to readily tune the material electronic and optical properties through wellestablished synthetic chemistry principles, have driven the development of thousands of organic materials. $^{1-6}$ To date, organic semiconductors have been exploited in a wide range of applications, from light-emitting diodes for displays and solid-state lighting, solar cells, fieldeffect transistors, all-optical switches, or biosensors, and represent already a $\$$ multi-billion market. $^{7-22}$

Advances in organic synthesis and molecular design, along with considerable improvement in the understanding of how to control the processing of these thin-film materials, have resulted in continued enhancement of device performance over the years. ${ }^{23-25}$ However, organic semiconductors still suffer from numerous drawbacks when compared to their inorganic counterparts. A key parameter in many of these applications is the ability to effectively transport charges, either holes or electrons, or both in the case of ambipolar materials. Efficient chargecarrier transport, along with aspects of the optical response, strongly depends on the electronic couplings among neighboring molecules or chain segments, factors that are directly contingent on the solid-state molecular packing arrangements. Hence, there is growing interest in how to design the chemical composition of molecules and polymers to dictate not only the electronic and optical response of the isolated molecules, but also the noncovalent interactions that direct molecular packing. ${ }^{26-38}$ 
Prime examples of how changes in chemical structure impact molecular packing and, in turn, the material electrical response can be found in the oligoacene family, a set of linearly fused aromatic hydrocarbons. If we take the examples of tetracene and its derivative rubrene $(5,6,11,12$-tetra[phenyl]tetracene $)$-the latter of which is discussed in more detail in section 3.2 of this chapter- ${ }^{39,40}$ we find that crystalline tetracene packs in the edge-to-face herringbone configuration typical of most oligoacenes; on the other hand, the tetraphenyl substitution along the long axis of the tetracene core in rubrene transforms the preferred packing configuration to one where the tetracene backbones are arranged in a slipped, face-to-face, often referred to as " $\pi$ stacked", configuration. This configuration in rubrene leads to substantial spatial overlap of the tetracene backbones and wavefunction overlap of the tetracene-based frontier $\pi$ orbitals, resulting in large intermolecular electronic couplings and record-high charge-carrier mobilities (up to 40 $\mathrm{cm}^{2} \mathrm{~V}^{-1} \mathrm{~s}^{-1}$ ) in single-crystal field-effect transistors; ${ }^{39-41}$ as a point of reference, electron mobilities in amorphous silicon range from $10^{-3}$ to $10^{1} \mathrm{~cm}^{2} \mathrm{~V}^{-1} \mathrm{~s}^{-1}, 42,43$ depending on sample preparation and doping. Chemical functionalization of rubrene, in attempts to improve crystal packing, has achieved varying success. Such results, as we will discuss, are in fact highly dependent on the noncovalent interactions that exist in these materials. ${ }^{44-47}$

Variations in processing can also be used to enhance the material charge-carrier transport properties. Recent advances in solution processing techniques, such as drop casting, spin coating, or meniscus-guided coating, have been developed with a view to direct the solid-state morphology of organic semiconductors. ${ }^{48}$ These advances will not be discussed here and the interested reader is directed to recent reviews on the subject. ${ }^{23-25}$ However, noncovalent interactions in the ink (i.e., solution) formulations of solution-processable semiconductors are 
critical; we will show some initial attempts to understand how such interactions in the solution environment can seed the development of the thin-film morphology of organic semiconductors.

To this point, we have discussed noncovalent intermolecular interactions in a rather general way. Building on previous Chapters in this book, see for instance Chapter 1, we now briefly reintroduce the key interactions, as these definitions will serve an essential role in the discussion that follows:

Electrostatic interactions arise from interactions among unperturbed charge distributions on neighboring molecules. The charge distributions on a molecule can be expressed in the form of a multipole expansion, i.e., monopole (charge), dipole, quadrupole, etc., starting with the lowestorder permanent multipole moment; the interactions arising from these multipoles include monopole-monopole, monopole-dipole, monopole-quadrupole, dipole-dipole, dipole-quadrupole, quadrupole-quadrupole, etc. It is important to bear in mind that such a multipole expression is valid only if the two monomers are separated by distances that are typically over $4 \AA$ (see Chapter 1 for further details on the convergence of the multipolar expansion). When the molecules are brought close together, the monomer charge densities start to overlap significantly, with the charge density on one molecule experiencing attraction from the nuclei of the other molecule. This increased electrostatic attraction results in an extra stabilization referred to as charge penetration. ${ }^{49-51}$ In many relevant organic semiconductors, the plane-to-plane distance is typically $<4 \AA ̊$; hence, significant charge penetration effects can be present.

Induction can be understood as the energy stabilization due to the electronic relaxation of the charge distribution on one molecule in response to the presence of another molecule; in other words, induction is the stabilization due to the induced electrostatic moments on one molecule 
owing to the presence of permanent multipole moments of the other. In the case of $\pi$-conjugated organic materials, the contribution from induction is generally small in magnitude, except when dealing with charged monomers.

Dispersion is dominant contributor to intermolecular stabilization in many $\pi$-conjugated molecular materials. Dispersion arises from instantaneous charge fluctuations (induced dipoles) in the electron density on each interacting molecule.

Exchange-repulsion, finally, is the consequence of Pauli's exclusion principle, which prevents two electrons [fermions] from occupying the same quantum state and therefore the same region of space. ${ }^{52,53}$ Exchange-repulsion physically balances the attractive forces, with the repulsion between electrons from adjacent molecules limiting the degree of contact.

The electronic and optical properties of organic semiconductors can be tuned by changing the respective contributions from these interactions as illustrated in Figure 1, and this ability to tune properties paves the way to the design of new functional materials with desired physicochemical properties. The relative importance of the noncovalent interactions strongly depends on the molecular chemical composition and architecture, with multiple terms often playing critical roles that ultimately determine intermolecular packing. In the following Sections, we begin by providing a brief overview of some of the chemical design principles that are used to direct the (opto)electronic characteristics of organic semiconductors. We then showcase examples where theory and computation have driven a deeper understanding of how chemical changes impact the preference for molecular packing configurations in the solid state, therefore enabling some of the considerations that are required to develop new generations of organic semiconductors. 


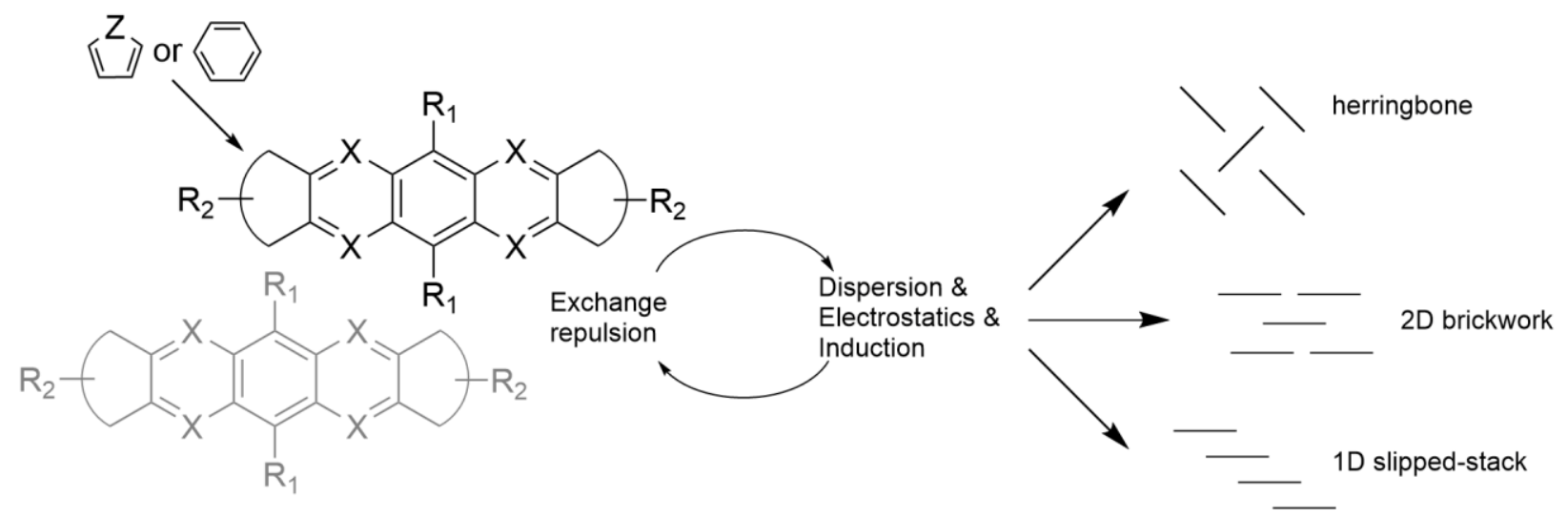

Figure 1. Using oligoacenes as a model, modifications to the length of the $\pi$-conjugated framework, substitutions (X) within the framework, or moieties substituted along the periphery $\left(\mathrm{R}_{1}\right.$ and $\mathrm{R}_{2}$ ) not only will affect the molecular redox and (opto)electronic characteristics, but also the nature and strength of the noncovalent intermolecular interactions (exchange repulsion, dispersion, electrostatics, and induction) that energetically drive certain molecular packing configurations in the solid state. Here, the nature and strength of the noncovalent interactions between two molecules - one in gray and one in black - can lead numerous ordered packing configurations, including herringbone, one-dimensional [1D] slipped-stack, or two-dimensional [2D] brickwork, to name but a few.

\section{Materials Design Motifs}

In this Section, we elaborate on some strategies that have been used to design $\pi$-conjugated molecular and polymeric materials. We begin with a very short overview of the connections between charge-carrier transport and molecular packing in the solid state. We then briefly describe how heteroatoms incorporated into the $\pi$-conjugated backbone and halogen atoms attached to the periphery of the backbone can influence molecular packing. We finally emphasize the importance of alkyl side-chains with respect to solubility, packing, and the resulting device performance. Admittedly, this overview is by no means exhaustive given the thousands of organic semiconductors that have been synthesized. 
We note that discussions centering on $\pi-\pi$ interactions are omnipresent in the organic semiconductor literature, as the building blocks of these materials contain a conjugated backbone, often with one or more aromatic rings. Quantum-chemical calculations with energy decomposition analysis (EDA) provide insight into defining these interactions, even though a complete physical picture still needs to be reached (see Chapter 2). ${ }^{50,51,54}$

2.1. Charge-Carrier Transport in Organic Semiconductors: Dependence on the Intermolecular Electronic Coupling

Given the dependence of the charge-carrier transport characteristics of organic semiconductors on the noncovalent interactions that in part define molecular packing in the solid state, it is not surprising that definitive models to describe these molecular-scale processes are still under development. Interested readers are referred to detailed reviews we have published on this topic. ${ }^{55-57}$ In general, charge-carrier transport in organic materials can be described in two limited regimes: band transport or thermally activated hopping. The band transport model is applicable to instances where the molecular wavefunctions delocalize over many sites (or molecules) in the solid-state material and form electronic bands; evidence for band transport has only been observed in highly pure molecular crystals. ${ }^{58}$ Band transport implies very strong intermolecular electronic couplings among nearest neighbors. Since these couplings directly depend on the wavefunction overlap of the relevant frontier molecular orbitals, molecular arrangements in the solid state (i.e., the relative intermolecular distances, orientations, and translations) have a tremendous influence on the magnitude of these electronic couplings.

In many instances, the weak noncovalent interactions in organic semiconductors, as opposed to the covalent bonds among atoms in inorganic (semi)conductors, result in relatively large 
intermolecular separations (greater than $3.2 \AA$ ) when compared to a covalent bond and large degrees of intrinsic disorder (related to thermal fluctuations) and extrinsic disorder (grain boundaries, impurities). These attributes, in turn, lead to wavefunction (or charge) localization on a single or just a few molecules. Then, the transport of charge carriers corresponds to a thermally activated hopping process, where the carriers move, or hop, from one molecule or chain segment to the next.

\subsection{Heteroatoms within the $\pi$-Conjugated Backbone}

Heteroatom substitution within the $\pi$-conjugated backbone has been widely used to alter the electronic characteristics of molecules and polymers. The impact of heteroatoms on these characteristics comes from changing the spatial extent of the $\pi$-electron cloud and the polarizability of the aromatic systems. As a result, the magnitudes of the interactions (especially when the molecule is made to become non-centrosymmetric) can be greatly affected, which in turn influences the preferred packing configurations when compared to their hydrocarbon counterparts. Sulfur ${ }^{59-68}$ and nitrogen ${ }^{69-71}$ atoms are widely substituted into the $\pi$-conjugated frameworks, with oxygen, selenium, and germanium also finding greater use. We provide later on a more detailed discussion of how heteroatom substitution influences the electronic coupling between adjacent molecules.

\subsection{Halogen Substituents on the $\pi$-Conjugated Backbone Periphery}

A number of halogen-substituted $\pi$-conjugated small molecules and polymers have garnered attention as efficient materials for thin-film transistors and solar cells, with the halogenation employed to alter the redox stability of organic semiconductors. The strong electron-withdrawing character of halogen atoms makes them particularly useful in the design of $n$-type 
semiconductors due to their ability to lower the reduction potential (i.e., increase the electron affinity). The electronegative nature of halogen atoms leads to molecules with very polar bonds. Halogen atoms decline in electron-withdrawing strength as one moves down the periodic table from fluorine to iodine. These polar bonds can play a considerable role in defining the nature of the noncovalent interactions that drive molecular packing. ${ }^{72-75}$

Fluorine is among the most widely used halogen atoms in organic semiconductors. Fluorination of aromatic rings can lead to a reversal of the characteristics of the $\pi$-electron density above and below on aromatic ring: perfluorinated aromatic rings lead to large, positive quadrupole moments, with positive poles above and below the rings (and negative poles in the plane of the nuclei), while the opposite is true for unsubstituted rings. ${ }^{76}$ Hence, upon fluorination, the drive to maximize the electrostatic interactions and reduce the exchange-repulsion term can lead to very different packing motifs. ${ }^{77}$ For example, appending fluorine atoms to pentacene converts the crystal packing from herringbone to a $\pi$-stacked motif (Figure 2). ${ }^{78,79}$ 
<smiles>c1ccc2cc3cc4cc5ccccc5cc4cc3cc2c1</smiles><smiles>Fc1c(F)c(F)c2c(F)c3c(F)c4c(F)c5c(F)c(F)c(F)c(F)c5c(F)c4c(F)c3c(F)c2c1F</smiles>
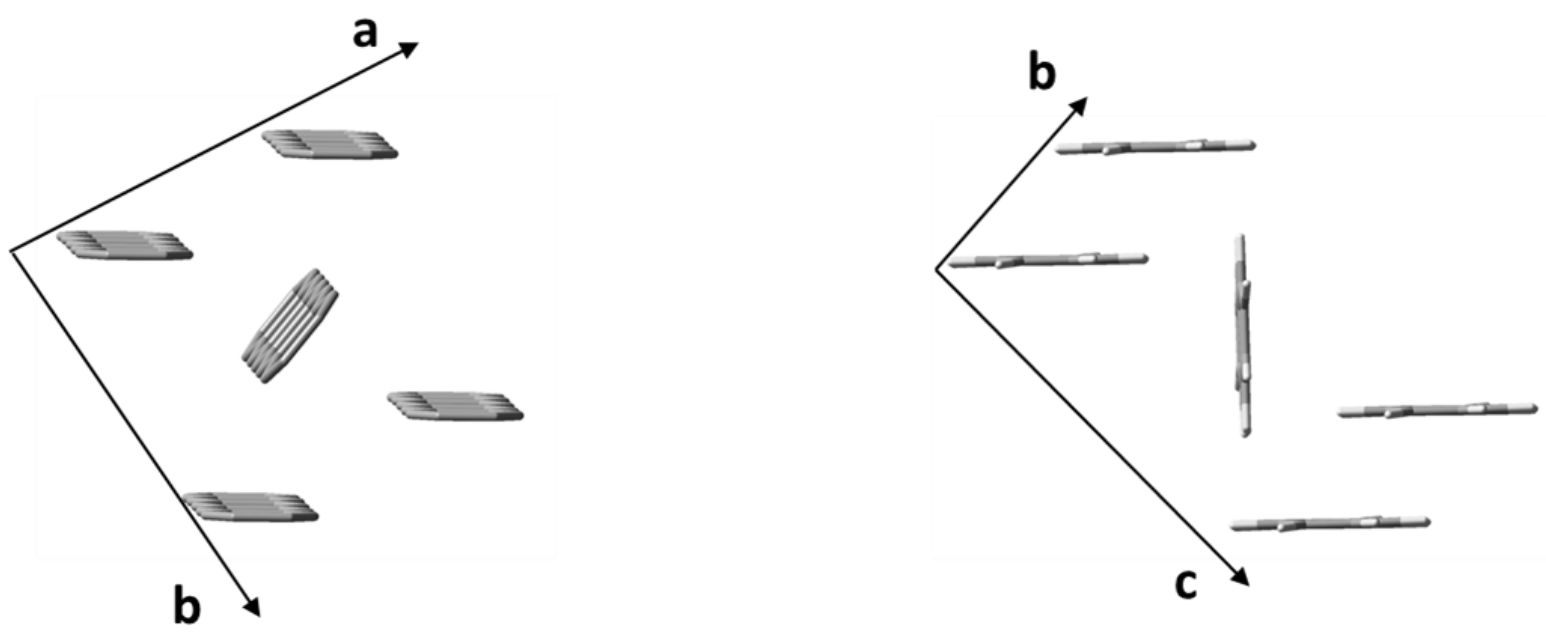

Figure 2. Chemical structures of pentacene (top, left) and perfluoropentacene (top, right) and illustrations of the crystal structure packing of pentacene ${ }^{78}$ (bottom, left) and perfluoropentacene ${ }^{79}$ (bottom, right).

The degree of halogenation can also play a role. For example, 5-chlorotetracene derivatives pack in a herringbone geometry whereas 5,11-dicholorotetracene and 5,6,11,12-tetracholorotetracene both arrange in face-to-face, slip-stacked configurations, with intermolecular distances of 3.48 and $3.56 \AA$, respectively. ${ }^{80,81}$ Anthony and co-workers synthesized fluorinated 6,13triisopropylsilylethynyl (TIPS)-pentacene derivatives, where the number of fluorine atoms on the peripheral rings shifts the intermolecular stacking distance; this distance goes from $3.43 \AA$ in the unsubstituted parent molecule to $3.36 \AA$ for the tetrafluoro structure to $3.28 \AA$ for the octafluoro structure. ${ }^{82}$ Perfluorination can also take place on the solubilizing alkyl side chains, which not only alters material miscibility but also influences the preferential charge-carrier transport in the 
molecular semiconductor; for instance, perfluoroalkyl functionalized oligothiophenes are $n$-type materials, whereas their alkyl cousins are $p$-type. ${ }^{83}$

Fluorine atom substitution has also been widely used to alter the solid-state morphology of $\pi$ conjugated polymers. Appropriate placement of fluorine atoms along the backbone can induce increased backbone planarity, which in turn can allow for tighter interchain packing, while the increased rigidity can enhance the charge-carrier transport properties along the chain. ${ }^{84-86}$ Fluorination has been carried out on a wide variety of building blocks used in the synthesis of polymers, including thiophenes, carbazoles, thienothiophenes, benzothiadiazoles, benzotriazoles, benzodithiophenes, indacenodithiophenes, and anthradithiophenes. ${ }^{87-96}$ Recently, we have shown in the case of poly(4,8-bis((2-ethylhexyl)oxy)benzo[1,2-b:4,5-b']dithiophene-thiophene $(\mathrm{PBDT}[2 \mathrm{H}] \mathrm{T})$ and its 3,4-difluoro-thiophene analog (PBDT[2F]T) polymers that fluorination induces greater planarity, enhanced chain stacking in the solid state, ${ }^{97}$ and larger inter-chain electronic couplings (Figure 3). Though halogenation is a widely used method to modify the redox and (opto)electronic properties of organic semiconductors, an a priori insight into how halogen substituents direct solid-state packing in organic semiconductors has still to be developed. ${ }^{89,95}$ 


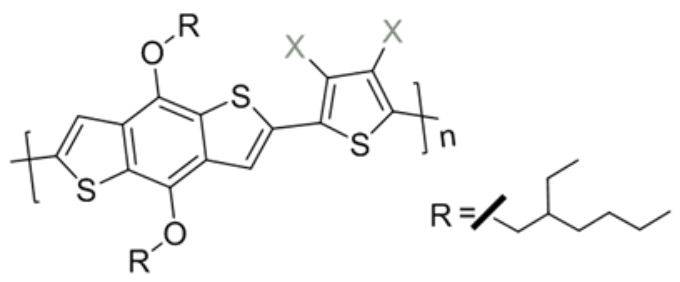

$$
\begin{array}{r}
X=H, P B D T[2 H] T \\
\text { F, PBDT[2F]T }
\end{array}
$$

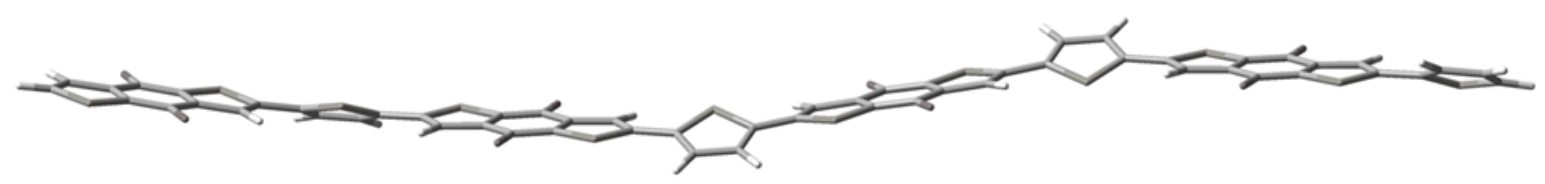

PBDT2[H]T

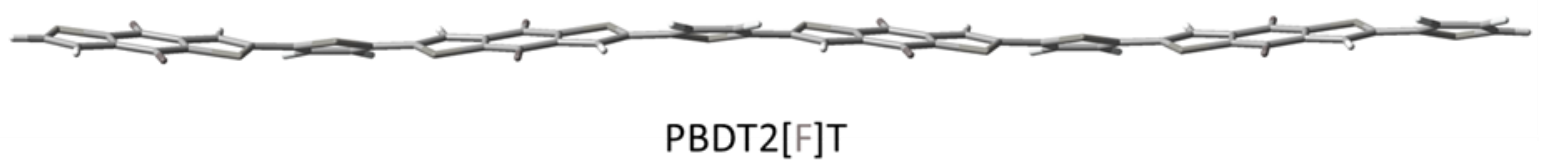

Figure 3: Chemical structures of the PBDT $[2 \mathrm{X}] \mathrm{T}$ polymers and illustrations of the backbone conformations of PBDT[2H]T and PBDT[2F]T oligomers. ${ }^{97}$

\subsection{The Role of Alkyl Chains: From Solubility to Steric Bulk}

Alkyl chains substituted on the periphery of the $\pi$-conjugated system are appended mainly to impart solubility and improve solution processing, but also greatly impact solid-state packing. ${ }^{37,98-100}$ Alkyl chains are (mostly) electronically inert. However, since they play an important role in determining the intermolecular packing, their presence influences the (opto)electronic properties of the organic semiconductor. Alkyl functionalization can help overcome the repulsive exchange interactions between $\pi$-conjugated moieties by shifting the balance between dispersion and exchange interactions. 
The idea of attaching bulky substituents has also been widely used to control the packing of small molecules. ${ }^{101-103}$ In particular, attaching bulky triisopropylsilyl (TIPS) groups via ethynyl linkers to the periphery of pentacene triggers the herringbone packing configuration of pentacene to transform into a 2D-brickwork packing arrangement. ${ }^{101}$ For benzothieno[3,2b]benzothiophene (BTBT), octyl chains appended along the molecular long axis leads to packing configurations that make BTBT a champion charge-carrier transport material in single-crystal field-effect transistors. ${ }^{104}$

For polymer-based materials, aliphatic alkyl side-chains appended to rigid polymer backbones modulate the solid-state morphology. ${ }^{37,98-100}$ In the case of PBDTTPD polymers (composed of alternating thieno[3,4-b]thiophene-pyrrole-4,6-dione and benzodithiophene units), crystallite formation and relative backbone orientations strongly depend on the pattern of alkyl chains along the polymer backbone. ${ }^{105}$ For instance, the PBDTTPD polymer with branched ethyl hexyl (EH) chains on the benzodithiophene (BTD) units and linear alkyl side-chain (C8) on the thieno[3,4c]pyrrole-4,6-dione (TPD) units forms crystallites with face-on orientation relative to substrate. On the other hand the PBDTTPD polymer with C8 substituents on BDT and EH substituents on TPD has an edge-on orientation relative to substrate (Figure 4). In addition, changes in the nature and positions of the alkyl chains have been shown to influence the efficiency of solar cells based on PBDTTPD polymers with fullerene acceptors: ${ }^{106,107}$ When TPD moieties carry linear alkyl side-chains and the BDT moieties carry branched side-chains, the fullerene molecules are expected to sit preferentially over the TPD segments of the polymer chain. This arrangement leads to much better solar cell performance compared to the polymer backbone where TPD units carry branched side-chains and BDT units carry linear side-chains. 


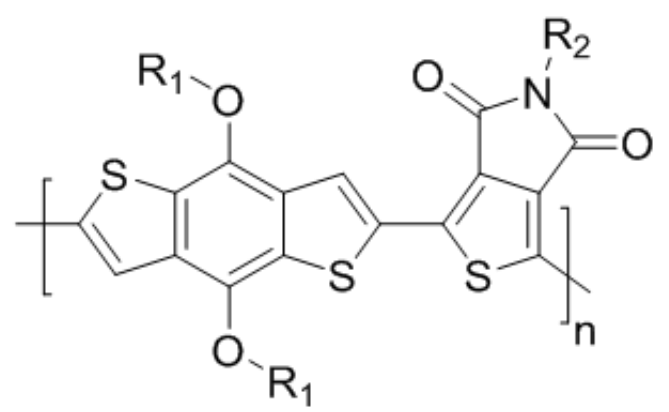

PBDTTPD $\left(\mathrm{C}_{14}-\mathrm{EH}\right)$ PBDTTPD $\left(\mathrm{C}_{14}-\mathrm{C}_{8}\right)$ PBDTTPD (EH-C 8$)$
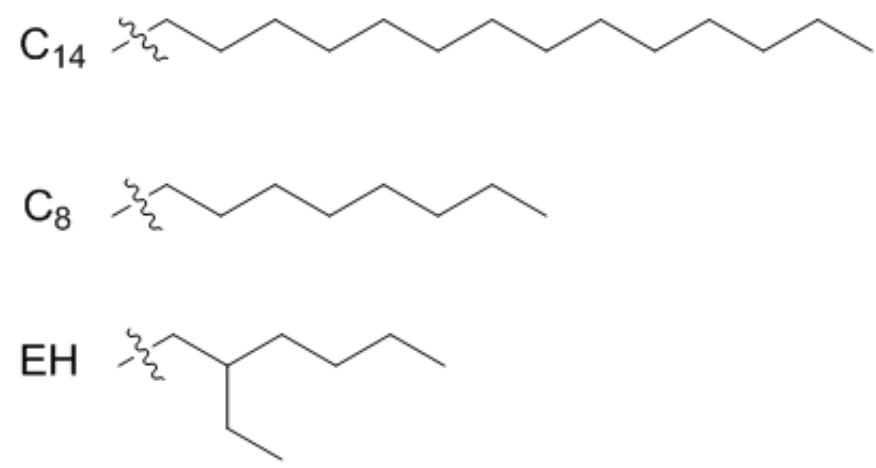

$\mathrm{R}_{1}=\mathrm{C}_{14} \quad \mathrm{R}_{2}=\mathrm{EH}$

$\mathrm{R}_{1}=\mathrm{C}_{14} \quad \mathrm{R}_{2}=\mathrm{C}_{8}$

$\mathrm{R}_{1}=\mathrm{EH} \quad \mathrm{R}_{2}=\mathrm{C}_{8}$

Figure 4. Chemical structures of PBDTTPD with the three side-chain patterns.

\section{Noncovalent Interactions in Molecular Materials}

As can be seen from the above discussion, there is a wide range of chemistries that can be used to tune the electronic and optical characteristics of organic semiconductors, in part by controlling the ways in which $\pi$-conjugated molecules and polymers pack in the solid state. We now turn our attention to the results of specific studies of $\pi$-conjugated molecules and polymers, both in neat (pure, single-component materials) and blend (multicomponent) forms, to show how computation and theory are driving the understanding required to a priori direct molecular packing and control materials-scale properties. 


\subsection{The Oligoacene Series}

To set a baseline for the discussion, we begin by examining the noncovalent interactions for the oligoacene series (i.e., benzene through hexacene, $n_{\text {rings }}=1-6$, Figure 5 ), ${ }^{51}$ as these fused aromatic systems can be considered as templates from which most molecular organic semiconductor materials are constructed. There exist a number of investigations exploring the nature and strength of the noncovalent interactions in these systems, which provide further reference: ${ }^{38,50,108-117}$ Grimme considered benzene through tetracene ${ }^{118}$ and Sherrill studied benzene through pentacene, ${ }^{119}$ with both studies focusing on a few, ideal dimer configurations, while Podeszwa and Szalewicz developed comprehensive potential energy surfaces for benzene through anthracene. ${ }^{120-122}$ Here, we will briefly discuss how the nature of the noncovalent interactions changes by starting with eclipsed cofacial arrangements and then considering longaxis translation and rotation about the long-molecular axis (Figure 5). 
<smiles>Cc1cccc(C)c1C</smiles>

$\mathrm{n}$

displacement along the long axis

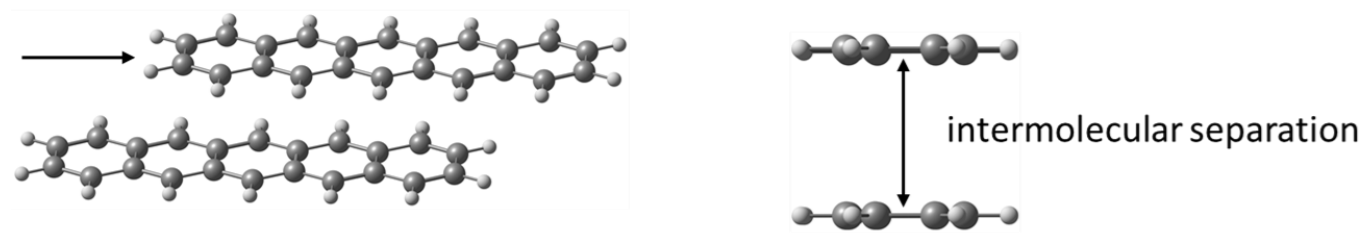

translation among stacked to T-shaped configurations
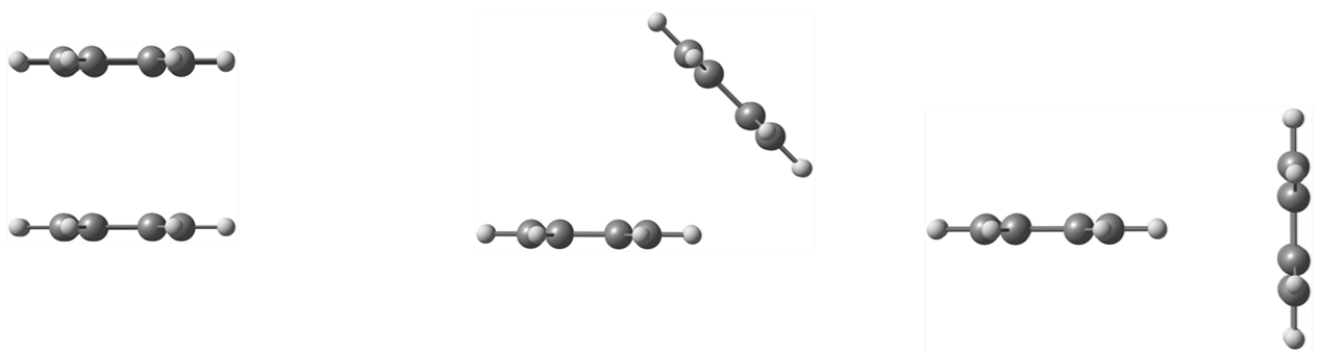

Figure 5. Chemical structures of linear oligoacenes, where $n$ is the number of fused rings: $n=0$ for benzene and $n=5$ for hexacene, and illustrations of pentacene dimers as a function of displacement along the long axis, varying intermolecular separation, and translation from stacked to T-shaped arrangements.

Using symmetry-adapted perturbation theory (SAPT) calculations at the SAPT0/jun-cc-pvdz level (here after simply denoted SAPT0; see Chapter 4 for further details), ${ }^{123}$ full potentialenergy surfaces for different oligoacene dimers under varying transformations were constructed. ${ }^{51}$ For cofacial stacking configurations, the total SAPT0 interaction energies fall off quickly, as expected, as the intermolecular distances move beyond $c a .3 .5 \AA{ }^{124}$ The exchange term decreases fastest, ${ }^{125}$ followed by the electrostatic and induction interactions, and then dispersion interactions. Notably, dispersion is the dominant attractive interaction at intermediate 
intermolecular separations (i.e., greater than $4.5 \AA$ ), though at even larger separations (i.e., larger than $7 \AA$ ) electrostatic interactions dominate; this is due to the $1 / R^{5}$ distance dependence of the quadrupole-quadrupole interactions compared to the approximately $1 / R^{6}$ distance dependence of dispersion.

Starting with cofacial dimers and slipping one molecule along the long axis (Figure 5) to create a two-dimensional potential energy surface reveals large fluctuations of the total interaction energies. ${ }^{51}$ The number of peaks and valleys of the fluctuations for the total interaction, exchange, and induction energies correspond with the number of fused rings (i.e., one maximum/minimum for benzene and five maxima/minima for pentacene), while the electrostatic energy displays maxima and minima opposite the other contributions. The interaction energy minima are found to correspond with staggered arrangements, where bridging carbon-carbon bonds are above the rings of the adjacent molecule.

At this stage we can make an important connection between the noncovalent interactions discussed above and the expected electronic characteristics of molecular organic semiconductors. ${ }^{38,126}$ Using a pentacene dimer with an intermolecular separation of $3.5 \AA$ as reference, one can readily draw direct connections between the exchange energy and the intermolecular electronic coupling, a key parameter used to describe the charge-carrier transport properties of organic electronic materials (as was mentioned in Section 2.1). As shown in Figure 6, a perfect co-facial stacking arrangement leads to the largest overlap of the highest-occupied molecular orbitals (HOMOs) on each molecule and, hence, the largest HOMO:HOMO electronic couplings ${ }^{127}$ and exchange repulsion (see Chapter 1). ${ }^{126,128}$ The electronic coupling and exchange repulsion generally decrease as one molecule is slipped along the long axis at fixed $3.5 \AA$ 
intermolecular separation. Both physical properties show an oscillatory pattern that correlates with the nodal pattern of the pentacene HOMO. It is useful to recall that the electronic coupling, as previously demonstrated for sexithienyl, ${ }^{127}$ goes to zero at varying molecular displacements, even though there remains considerable spatial overlap of the pentacene backbones. On the other hand, the exchange-repulsion energy in these systems remains large in magnitude for all displacements considered at the $3.5 \AA$ intermolecular separation, which arises from the fact that the exchange energy is determined from the overlap among all occupied orbitals, and is dominated by the electrons in the $\sigma$ framework.

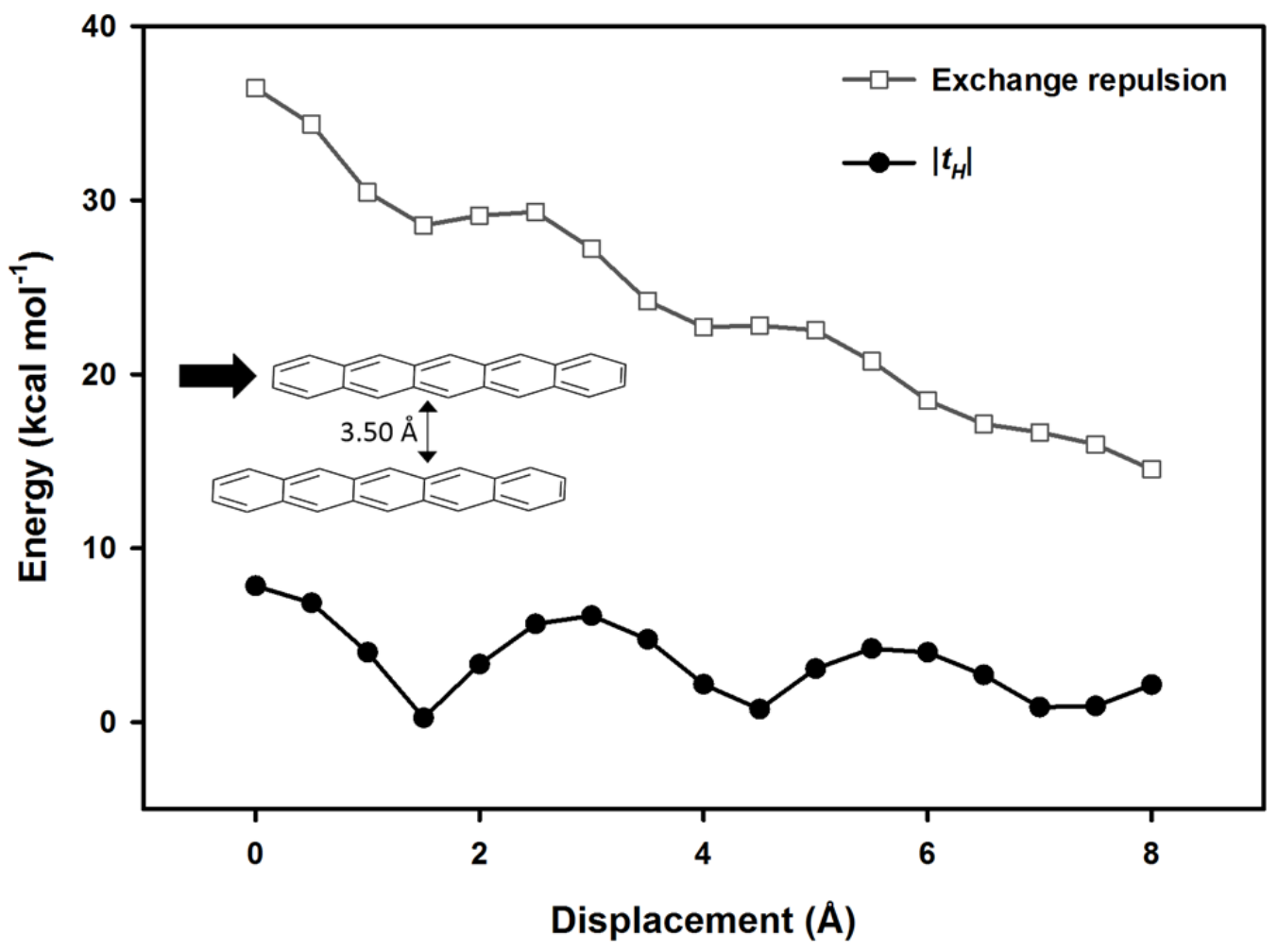

Figure 6. Intermolecular electronic couplings ( $t_{H}$, closed circles), determined from the absolute value of the HOMO:HOMO overlap at the B3LYP/cc-pVDZ level of theory, and exchangerepulsion energies (open squares), determined at the SAPT0/jun-cc-pVDZ level of theory, for a pentacene dimer with an intermolecular separation of $3.5 \AA$. Starting from a perfectly cofacial configuration, the top molecule is gradually displaced along the molecular long axis. 
Concurrent consideration of increased intermolecular separation and long-axis translation leads to fairly complex three-dimensional surfaces.$^{51}$ For benzene, there is only one minimum in the potential energy surface, while for tetracene and beyond, multiple minima in the potential energy surfaces become apparent, in line with the two-dimensional potential energy surfaces; these extra minima are even more apparent for the longer oligoacenes (e.g., hexacene). For each of these situations, the low energy structures correspond to staggered co-facial arrangements that are reminiscent of TIPS-pentacene, where the bridging carbon-carbon bonds of one molecule are interacting with the face of the fused rings on the adjacent molecule. ${ }^{101,102}$ While it is generally accepted that the TIPS groups dominate the packing, the non-bonded pentacene interactions act to fine tune the packing to result in the staggered, parallel-displaced arrangement (including a degree of slip along the short axis that is not considered here) of the $\pi$-conjugated systems.

In addition to long-axis translation, it also of interest to explore the impact of rotating one of the molecules in the dimer, as it provides a preview of the expected intermolecular interactions observed in the herringbone packing configurations found for oligoacenes in the solid state. ${ }^{51}$ Starting with rotation of one of the co-facial molecules by either $45^{\circ}$ or $90^{\circ}$ (T-shaped) and adjusting the intermolecular separation, it is found that the $45^{\circ}$ dimer is the least stable configuration considered for all acenes in the series, though as the acene length increases, the most stable configuration changes. For benzene, the T-shaped dimer is the most stable, with $45^{\circ}$ and eclipsed dimers being approximately equally unfavorable; in anthracene, the eclipsed dimer is intermediate of the $45^{\circ}$ and $90^{\circ}$ dimers, while for pentacene the eclipsed dimer is slightly more stable than the $90^{\circ}$ dimer. Rotation of the one of the molecules in the dimer from $0^{\circ}$ to $90^{\circ}$ at fixed intermolecular separations (Figure 5) reveals that for all systems the evolution of the total intermolecular interaction energy follows qualitatively those of exchange and electrostatics; 
minima are found at $20^{\circ}$ and $90^{\circ}$, with a maximum at about $60^{\circ}$ (i.e., exchange is more repulsive when electrostatics are more attractive). Here, electrostatics are dominated by charge penetration at the intermolecular distances considered; thus, both exchange and electrostatics are dependent on the extent of orbital overlap with their contributions being of opposite sign. We note that the intermolecular angles found in the oligoacene crystal structures (approximately $50^{\circ}$ to $80^{\circ}$ depending on the oligoacene) lie near the least stable intermolecular interaction energies. This points to a limitation in the current analysis, suggesting that important terms are not fully accounted for in a two-body SAPT0 approach; ${ }^{129-132}$ thus, models aiming to predict crystal packing need to go beyond such approaches. ${ }^{133}$

The analysis of the non-covalent interaction energies of oligoacene dimers reveals the complexity of molecular crystal engineering, with the small energy differences among rather different configurations providing a clear picture of why polymorphism is such a common phenomenon in these systems. ${ }^{51} \mathrm{We}$ now turn to studies on functionalized oligoacenes to highlight how chemical substitution can impart novel molecular packing in the solid state and, in turn, electronic properties.

\subsection{Rubrene: Solid-State Structure Determined by a Balance of Intramolecular and Intermolecular Noncovalent Interactions}

As noted in the Introduction, rubrene (labeled 1 in Figure 7) is an exemplar molecular organic semiconductor in terms of measured charge-carrier transport characteristics. It also provides an important example of how chemical substitution can impact in significant ways the solid-state molecular packing not only at the intermolecular level but also at the intramolecular level. For rubrene, large hole mobilities (as high as $40 \mathrm{~cm}^{2} \mathrm{~V}^{-1} \mathrm{~s}^{-1}$ ) 39 arise from strong intermolecular 
electronic couplings ${ }^{128,134-136}$ that are a result, in part, of the tetracene backbones being planar in the solid state. ${ }^{137,138}$ Figure 7 illustrates that the tetracene backbone in rubrene is substituted along the periphery by four phenyl groups. Electronic-structure calculations on isolated rubrene molecules show that these phenyl rings make the tetracene backbone of an isolated rubrene molecule preferentially twist $\left(\sim 40^{\circ}\right),{ }^{139-142}$ a result confirmed by experimental evidence of twisted conformations both in solution ${ }^{44,140}$ and in thin films. ${ }^{139,143}$ Hence, for future materials design, there is interest in understanding why the tetracene backone twists when rubrene is isolated and ${ }^{66}$ what intermolecular interactions in the solid state might lead to the planarization of the backbone. ${ }^{126}$

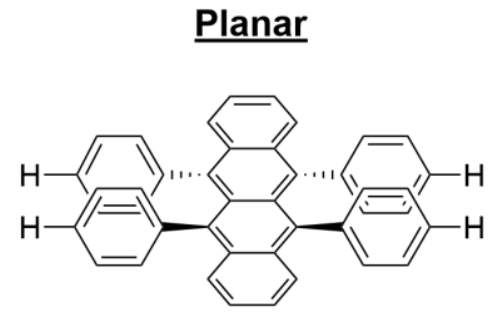

\section{Twisted}

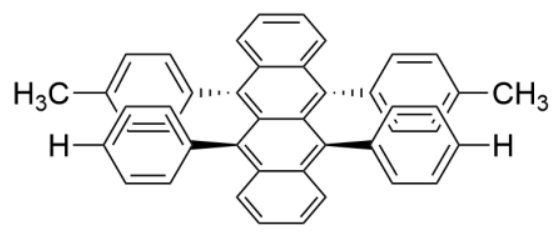

2

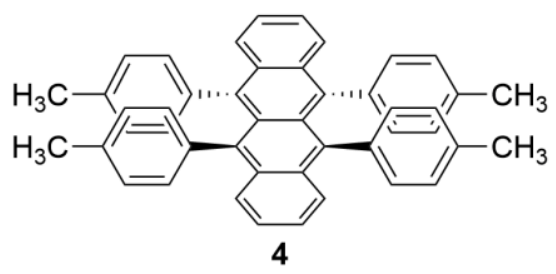

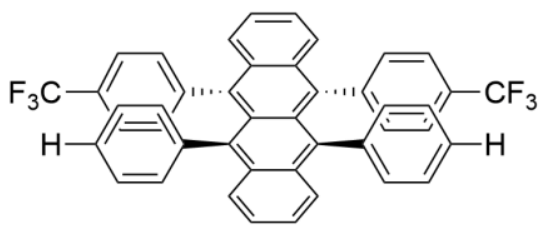

3

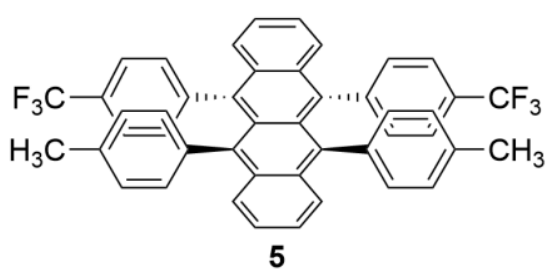

Figure 7. Chemical structures of rubrene $\mathbf{1}^{144}$ and representative rubrene derivatives $\mathbf{2}-\mathbf{5} .^{46} \mathbf{1}, \mathbf{3}$, and $\mathbf{5}$ possess planar tetracene backbones in their reported crystal structures, while $\mathbf{2}$ and $\mathbf{4}$ are twisted. 
To explore these questions, a large series of rubrene derivatives were studied, five of which are shown in Figure $7 .{ }^{126}$ For all rubrene derivatives considered, rubrene conformations with a twisted tetracene core (with twists of 30 to $40^{\circ}$ in a helical-like fashion) are favored by $c a .2$ to 4 $\mathrm{kcal} \mathrm{mol}^{-1}$ over rubrenes where the tetracene backbone is constrained to be planar; these results are consistent with previous theoretical studies. ${ }^{44,139-142}$ For an isolated tetracene molecule, the planar conformation is nearly $5 \mathrm{kcal} \mathrm{mol}^{-1}$ more stable than conformations with twists similar to those in rubrene; ${ }^{145}$ hence, the tetracene backbone finds itself in a highly unfavorable conformation in rubrene. The nearly equivalent stabilization induced by twisting the tetracene backbone across the series indicates that the exact nature and positions of the substituents on the phenyl rings have only a small effect on the energetics of the molecular structure.

For isolated rubrene molecules, it is noncovalent intramolecular interactions between phenyl groups on the same side of the molecule that are responsible for the twisting. The phenyl pairs anchored to the central rings of the tetracene backbone are constrained to an intermolecular separation (based on interatomic distances) of approximately $3 \AA$ at their closest point of contact.

To explore the impact of this close contact, phenyl pairs were extracted from the rubrene structures (with the broken carbon-carbon bond replaced by a carbon-hydrogen bond) and the noncovalent intermolecular interactions of the phenyl pair evaluated at the SAPT0 level of theory. The analysis reveals that, to minimize the unfavorable exchange interactions at such a close distance, the phenyl rings move away from the co-facial arrangement of a perfect $D_{2 h}$ conformation, resulting in a torque in the tetracene backbone. The repulsive exchange energy in these phenyl pairs is $c a .+20$ to $+23 \mathrm{kcal} \mathrm{mol}^{-1}$, an energy that is nearly twice as large as the dispersion and three times as large as the electrostatic energies. While these relative values are 
consistent with those for the benzene dimers at $3 \AA$, they are in absolute values about half as large as those for the fully eclipsed $\left(\mathrm{D}_{2 \mathrm{~h}}\right)$ benzene dimer at $3 \AA .^{50}$

Given that rubrene wants to maintain a twisted tetracene core, the question arises as to why some rubrene derivatives pack with a planar tetracene core in the solid state. The answer to this question is complex, and there remain many issues to address to provide a full understanding. However, a key outcome of the SAPT0 investigation is that interactions both within the plane, where the tetracene cores overlap, and between these planes, where the phenyl rings directly interact, assist in the planarization of the tetracene core. ${ }^{126}$

3.3. Azapentacenes and Benzodithiophene as Model Systems to Investigate the Impacts of Heteroatom Substitution

The query now turns to how heteroatom substitution impacts noncovalent interactions in these materials and, in turn, the intermolecular electronic couplings. We start with a series of azapentacenes (Figure 8) to explore how nitrogen-atom substitution modulates the characteristics set forth by the oligoacenes. ${ }^{38}$ SAPT0 results for co-facial configurations at an intermolecular distance of $3.50 \AA$ generally confirm predictions from experiment and previous calculations on small model systems: Introducing nitrogen into the $\pi$-conjugated backbone reduces the exchange repulsion, with each successive substitution of two nitrogen atoms for two -CH groups (e.g., from pentacene to the diazapentacenes and tetraazapentacenes) lowering the exchange repulsion by 2.5 to $4.7 \mathrm{kcal} \mathrm{mol}^{-1}$. The reduction in the exchange repulsion (arising from smaller wavefunction overlap with increasing nitrogen content) is evidence for contracted electron density in the $N$-heteropentacenes. The dispersion and electrostatic energies are also generally reduced with nitrogen substitution. Hence, even though the molecules in the model series are iso- 
electronic and the same intermolecular separations are considered, the nature and strength of the intermolecular interactions vary in important ways with the rather subtle changes in chemistry.<smiles>c1ccc2cc3cc4cc5ccccc5cc4cc3cc2c1</smiles><smiles></smiles><smiles>c1cnc2cc3cc4cc5nccnc5cc4cc3nc2c1</smiles><smiles>c1ccc2nc3cc4cc5ccccc5nc4cc3cc2c1</smiles><smiles>c1cnc2cc3cc4cc5nccnc5cc4cc3cc2n1</smiles><smiles>c1ccc2cc3nc4cc5ccccc5cc4nc3cc2c1</smiles><smiles>c1ccc2cc3cc4nc5nccnc5nc4cc3cc2c1</smiles><smiles>c1cnc2cc3cc4cc5ncccc5cc4cc3cc2c1</smiles><smiles>c1ccc2nc3cc4nc5ccccc5nc4cc3nc2c1</smiles><smiles>c1cnc2cc3cc4cc5cccnc5cc4cc3cc2c1</smiles>

Figure 8. Chemical structures of pentacene (left) and select diazapentacenes (middle) and tetraazapentacenes (right).

Likewise, the electronic couplings are found to decrease with nitrogen substitution, with the exact decrease depending on the specific location of functionalization and orientation of the molecules in the azapentacene pairs. ${ }^{38}$ As with pentacene, the azapentenacenes show the expected correlation between exchange-repulsion and intermolecular electronic coupling, as both factors depend on the degree of wavefunction overlap. The scale of these energies, however, is considerably altered by the preferred molecular orientation. Due to dipole moments that arise from asymmetric nitrogen substitution, increased electrostatic interactions favor dimer 
configurations with less spatial overlap among the majority aromatic hydrocarbon components of the molecular structures, resulting in smaller electronic couplings.

Positional disorder of thiophene atoms in thienoacenes, and specifically in anthradithiophenes (ADT), has been related to the varying charge-carrier transport characteristics of the molecular materials derived from these systems. ${ }^{146,147}$ As thin-films of the molecular organic semiconductor materials are generally disordered, pathways of consistent molecular alignments that mitigate the impact of charge trapping sites (e.g., defect sites within the material that lead to electronic disorder) are necessary to provide efficient charge-carrier transport conduits through the active layer. From a materials design standpoint, it is important to realize that the substitution of thiophene into an acene structure can lead to an isomeric mixture of products. ${ }^{146,148-150}$ In addition to isomeric purity, molecular symmetry can play a role. For molecules with low degrees of symmetry, adjacent molecules can pack in a disordered manner despite the molecules being isomerically equivalent. For isomerically pure ADTs, the anti isomer shows the lowest degree of thiophene positional disorder in the experimental crystal structures, ${ }^{146,149}$ which could lead to more homogenous charge-carrier transport pathways and offers a plausible explanation for the larger hole mobilities determined from transistor studies.

To discuss the effects of disorder in a more descriptive manner, the concept of the disordermer a form of intermolecular dissimilarity between regiochemically identical molecules due to disorder in the crystalline state - was recently introduced. ${ }^{147}$ Figure 9 demonstrates this concept with anti benzodithiophene (BDT); we note that the syn configuration of BDT is one in which the sulfur atoms in the thiophene rings lie on the same side of the molecule. In solution, the two BDT configurations drawn at the top of Figure 9, due to their averaged motion, are equivalent. However, in the solid state, the orientational disorder of the gray molecules can be seen with 
respect to the black BDT molecules. Since the positions of the molecules in the solid state are fixed (i.e., translation and rotational motion of the molecule are prevented), there appears two disordermers.

solution<smiles>c1cc2cc3sccc3cc2s1</smiles>

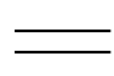<smiles>c1cc2cc3sccc3cc2s1</smiles>

solid state
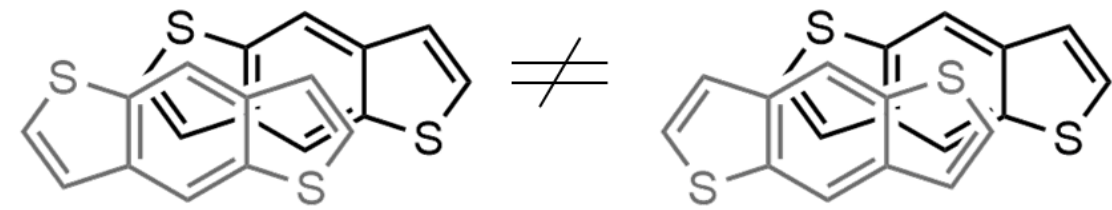

Figure 9. Illustration of the disordermer concept for anti benzodithiophene (BDT). The blue and green structures in solution (top row) are equivalent due to their averaged molecular motions. In the solid state (bottom row), the molecular positions are fixed so that the molecules cannot reorient. In this case, there are two possible disordermers with respect to the black BDT molecules.

SAPT0 and density functional theory (DFT) calculations of syn and anti BDT disordermers reveal important differences in the noncovalent interactions and subsequent intermolecular electronic couplings as a function of the disordermer. ${ }^{147}$ In general, contacts where sulfur atoms are in close proximity are less energetically favorable than those where the sulfur atoms are further away from each other, due to large exchange-repulsion energies, while the electronic couplings tend to be larger when direct sulfur contacts are present. These results can be related back to the characteristics of isomerically pure ADT molecular materials. Analysis of the ADT crystal structures ${ }^{146,148-150}$ shows that the dominant disordermers tend to minimize sulfur contacts 
among nearest neighbors: The anti ADT isomer is able to sustain a pattern of minimal sulfur contacts in the solid state, while the symmetry of the syn ADT isomer does not allow this to occur, resulting in the presence of much more disorder in the crystal; the larger degree of disorder in syn ADT appears to be the reason behind the worse transistor performance when compared to transistors using anti ADT as the active layer. Thus, while heteroatoms are widely used in the design of organic semiconductors, making seemingly subtle changes in the chemistry of the $\pi$-conjugated backbone through heteroatom substitution to alter the redox and (opto)electronic characteristics, can have wide ranging materials implications.

3.4. Noncovalent Interactions in Fullerenes and Polymer:Fullerene Blends: Implications for Processing and Solar Cells

To this point, we have considered only neat, or pure, materials. However, ink formulations from which organic semiconductors are deposited are generally multicomponent systems. One of the more promising applications for organic semiconductors, as solar cells, requires a blend of at least two materials in the active layer. Hence, there is a need to understand how variations in noncovalent interactions can impact multicomponent systems across the field of organic electronics.

We start with an analysis of fullerenes, namely $\mathrm{PC}_{61} \mathrm{BM}$ (phenyl- $\mathrm{C}_{61}$-butyric acid methyl ester, Figure 10), in solution. ${ }^{151}$ In combination with chlorinated solvents, high-boiling point solvent additives are typically used during the solution processing of organic photovoltaic (OPV) activelayer formulations to impact the morphological / topological features of the bulk heterojunction (BHJ) multicomponent thin film. However, little is known as to how and why these additives affect solubility. 

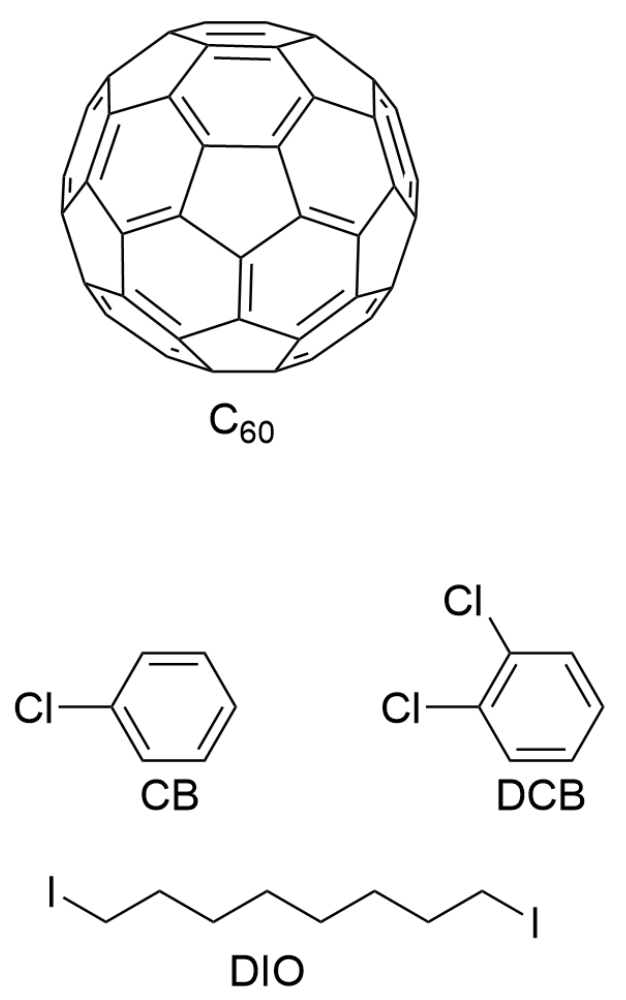

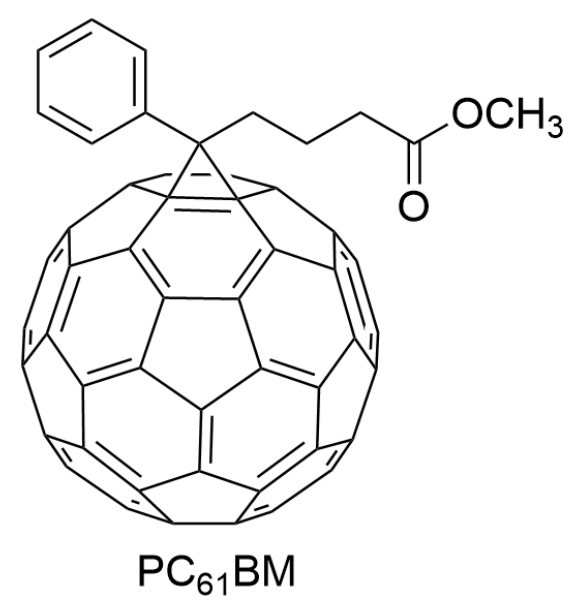<smiles>N#[Pb]c1cccc2ccccc12</smiles><smiles>N#Cc1ccccc1Cl</smiles>

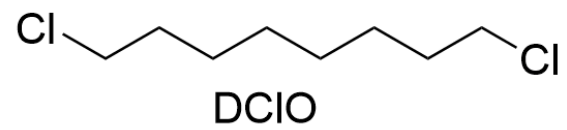

Figure 10. Chemical structures of $\mathrm{C}_{60}, \mathrm{PC}_{61} \mathrm{BM}$ (phenyl- $\mathrm{C}_{61}$-butyric acid methyl ester), chlorobenzene (CB), o-dichlorobenzene, naphthalene (NAP), 1-chloronaphthalene $(1 \mathrm{CN}), 1,8$ diiodooctane (DIO), and 1,8-dichlorooctane (DClO).

The unsubstituted fullerene, $\mathrm{C}_{60}$, can be used as a baseline to understand $\mathrm{PC}_{61} \mathrm{BM}$ solubility. ${ }^{151}$ Potential energy surfaces were computed at the SAPT0 level of theory for $\mathrm{C}_{60}$-solvent (either chlorobenzene $[\mathrm{CB}]$ or $o$-dichlorobenzene $[\mathrm{DCB}])$ and $\mathrm{C}_{60}$-additive interactions. The results indicate that the $\mathrm{C}_{60}-\mathrm{DCB}$ interaction is $0.5 \mathrm{kcal} / \mathrm{mol}$ more stable than $\mathrm{C}_{60}$ with $\mathrm{CB}$, a consequence of the extra chlorine atom in DCB, which increases each of the three stabilizing (electrostatic, dispersion, induction) terms. For the $\mathrm{C}_{60}$-additive interactions, the strengths of the interactions follow: 1-chloronaphthalene $(1 \mathrm{CN})>$ unsubstituted naphthalene $(\mathrm{NAP})>1,8-$ 
dichlorooctane (DClO). Though $\mathrm{DClO}$ lacks $\pi$-character, when compared to DCB (with two chlorine atoms), the two systems show similar dispersion interactions with $\mathrm{C}_{60}$, while the centrosymmetric nature of $\mathrm{DClO}$ (and the resulting zero dipole moment) leads to limited electrostatic and induction interactions. The exchange-repulsion interactions increase for the $\mathrm{C}_{60^{-}}$ solvent interactions in identical ways for both $\mathrm{CB}$ and $\mathrm{DCB}$, while the increase is steeper for $1 \mathrm{CN}$ and NAP due to greater number electrons (including the aromatic $\pi$ electrons) that increase the exchange term.

For the $\mathrm{PC}_{61} \mathrm{BM}$-solvent interactions, the repulsive wall of the potential energy surface rises at a smaller interaction distance $(6.4 \AA)$ when compared to the $\mathrm{C}_{60}$-solvent interactions $(6.8 \AA)$. These differences are indicative of effects due to the fullerene adduct, and correlate with the higher solubility of $\mathrm{PC}_{61} \mathrm{BM}$ in these solvents, especially for $\mathrm{PC}_{61} \mathrm{BM}$ in $\mathrm{CB}$. Turning to the $\mathrm{PC}_{61} \mathrm{BM}-$ additive interactions, while $\mathrm{DClO}$ reveals a tendency for much weaker binding with $\mathrm{PC}_{61} \mathrm{BM}$ when compared to $\mathrm{CB}$ and $\mathrm{DCB}, 1 \mathrm{CN}$ and NAP interact rather strongly and at similar center-ofmass distances, results that are in line with those of $\mathrm{C}_{60}$. Across the solvents and additives considered, the intermolecular interactions tend to be stronger with $\mathrm{PC}_{61} \mathrm{BM}$ when compared to $\mathrm{C}_{60}$, a factor directly attributable to the adduct.

The SAPT0-derived noncovalent intermolecular interactions can be related, in part, to the widely used Hildebrand and Hansen solubility parameters. ${ }^{151-153}$ The Hildebrand parameter $\left(\delta_{\mathrm{T}}\right)$ corresponds to the square-root of the cohesive energy density, i.e., the intermolecular interaction energy in the solid state per unit volume. Hansen further separated the Hildebrand parameter into three intermolecular contributions (which are referred to as the Hansen solubility parameters): dispersion $\left(\delta_{\mathrm{D}}\right)$, Coulombic or dipole-dipole $\left(\delta_{\mathrm{P}}\right)$, and hydrogen-bonding $\left(\delta_{\mathrm{H}}\right)$ interactions $)$. Moreover, we have shown with molecular dynamics (MD) simulations using the OPLS-AA 
force field the ability to evaluate Hildebrand and Hansen parameters for $\mathrm{PC}_{61} \mathrm{BM}, \mathrm{PC}_{71} \mathrm{BM}$ (phenyl- $\mathrm{C}_{71}$-butyric acid methyl ester), DCB, and CB with good semi-qualitative agreement with experiment. In this context, the comparisons made here are qualitative, and provide an upper bound for the noncovalent intermolecular interactions that are critical to determining the solubility parameters.

Having gained insight into how the substituent appended to the fullerene cage can affect solubility, we now describe how the substituent on the fullerene can impact interactions with $\pi$ conjugated polymers and, in turn, the mechanical properties as considered through the cohesive energies (i.e., a measure of the noncovalent interactions holding together molecular and polymerbased materials in the solid state). Here, we focus on two fullerenes $-\mathrm{PC}_{61} \mathrm{BM}$ and ICMA (indene- $\mathrm{C}_{60}$-mono adduct, Figure 11$)$ - and the widely studied $\pi$-conjugated polymer poly(3hexylthiophene) (P3HT).

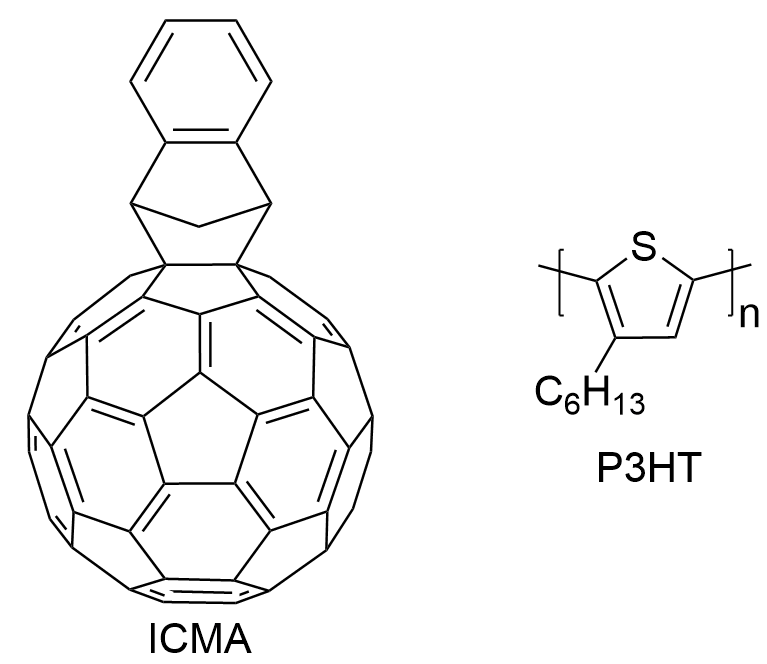

Figure 11. Chemical structures of ICMA (indene-C60-mono adduct) and poly(3-hexylthiophene) (P3HT). 
The Hildebrand parameters computed from MD simulations using the OPLS-AA force field for $\mathrm{PC}_{61} \mathrm{BM}\left(10.7[\mathrm{cal} / \mathrm{cc}]^{1 / 2}\right)^{154}, \operatorname{ICMA}\left(10.0[\mathrm{cal} / \mathrm{cc}]^{1 / 2}\right)$, and P3HT $\left(9.2[\mathrm{cal} / \mathrm{cc}]^{1 / 2}\right)$ compare very favorably with experimental data. ${ }^{155,156}$ Decomposition of the total interaction energy into dispersive, electrostatic, and hydrogen-bonded interactions yields the Hansen miscibility parameters: ${ }^{153}$ The dispersion parameters ${ }^{153}\left(\delta_{\mathrm{D}}\right)$ are identical for the two fullerenes $(9.8$ and 9.9 $[\mathrm{cal} / \mathrm{cc}]^{1 / 2}$ for ICMA and $\mathrm{PC}_{61} \mathrm{BM}$, respectively). The contribution from electrostatic interactions, however, is reduced in ICMA when compared with $\mathrm{PC}_{61} \mathrm{BM}$, as the combined hydrogen-bond plus electrostatic term $\left(\delta_{\mathrm{P}}+\delta_{\mathrm{H}}\right)$ is $4.0(\mathrm{cal} / \mathrm{cc})^{1 / 2}$ in $\mathrm{PC}_{61} \mathrm{BM}$ and only $2.0(\mathrm{cal} / \mathrm{cc})^{1 / 2}$ in ICMA. The same term in $\mathrm{P} 3 \mathrm{HT}$ is $3.0(\mathrm{cal} / \mathrm{cc})^{1 / 2}$. The Hansen parameters arising from the simulations suggest that the larger $\left(\delta_{\mathrm{P}}+\delta_{\mathrm{H}}\right)$ term in $\mathrm{PC}_{61} \mathrm{BM}$, a direct function of the polar nature of the side chain, could lead to a smaller driving force for mixing with P3HT when compared to ICMA, even though both fullerene derivatives lie within the solubility sphere of P3HT. ${ }^{157}$ This hypothesis is consistent with the experimental observation of the formation of fullerene clusters in P3HT:PC ${ }_{61} \mathrm{BM}$ mixtures ${ }^{158,159}$ and the improved miscibility of P3HT:indene-C $\mathrm{C}_{60}$-bis-adduct (ICBA) blends when compared to P3HT:PCBM. ${ }^{159}$

Turning to the mechanical properties of the pure materials, the calculated uniaxial elongation/tensile moduli for an elastic material, a measure of stiffness for the substituted fullerenes, are determined to be $8.1 \pm 0.01 \mathrm{GPa}$ for $\mathrm{PC}_{61} \mathrm{BM}$ and $8.3 \pm 0.01 \mathrm{GPa}$ for ICMA; the 0.2 GPa larger modulus for ICMA is a function of the shorter adducts that result in closer packing and higher density. ${ }^{160}$ While the differences in tensile modulus might at first appear to be minor, extremely small error bars suggest that this is a notable difference. The cohesion energy obtained from MD deformation simulations for ICMA is $0.36 \pm 0.05 \mathrm{~J} \mathrm{~m}^{-2}$, a result that is somewhat larger than the experimental value $\left(0.23 \mathrm{~J} \mathrm{~m}^{-2}\right)^{160}$ notably, ICMA clusters are formed 
during film formation from deposition with chlorobenzene, the solvent used in the corresponding experimental investigation, which could reduce the film cohesion measured experimentally. ${ }^{161}$ The calculated cohesion energy for $\mathrm{PC}_{61} \mathrm{BM}$ is $0.50 \pm 0.05 \mathrm{~J} \mathrm{~m}^{-2}$, a result that is in very close agreement with the experimental result of $0.51 \pm 0.07 \mathrm{~J} \mathrm{~m}^{-2}{ }^{160}$ These results provide confidence that the fullerene intermolecular interactions are well-described by the force field.

Deformation simulations, where the simulation box was elongated in one direction to induce strain on the system, were carried out at the MD level for pure P3HT and 1:1 weight-percent mixtures of P3HT:PC ${ }_{61} \mathrm{BM}$ and P3HT:ICMA for 50-monomer $(8.3 \mathrm{kDa})$ and 200-monomer $(33.2 \mathrm{kDa})$ P3HT. The pure polymer simulations, as expected, show very different behavior, with the higher-molecular-weight polymer able to handle more strain, a result directly attributable to the presence of substantial entanglements in the higher-molecular-weight system. ${ }^{160}$ The addition of the fullerene derivatives to low-molecular-weight P3HT results in no plateau with strain. In contrast, the 200-monomer P3HT:fullerene blends do show a stress plateau past 100\% strain. For ICMA:P3HT mixtures, a slightly larger stress is observed at strains greater than $200 \%$, indicative of a greater degree of entanglements in this blend compared to the $\mathrm{P} 3 \mathrm{HT}: \mathrm{PC}_{61} \mathrm{BM}$ mixtures. Upon elongation, due to stronger intermolecular interactions, P3HT:ICMA mixtures tend to form thick, inter-mixed fibrillar structures, in contrast to independent P3HT fibrils with fewer bound $\mathrm{PC}_{61} \mathrm{BM}$ molecules in the case of $\mathrm{P} 3 \mathrm{HT}: \mathrm{PC}_{61} \mathrm{BM}$ mixtures. ${ }^{160}$ Moreover, simulations of polymer/fullerene bilayers show that fracture tends to occur within the ICMA layer for the P3HT/ICMA bilayer, whereas the fracture occurs at the interface for a $\mathrm{P} 3 \mathrm{HT} / \mathrm{PC}_{61} \mathrm{BM}$ bilayer. Hence, relatively modest changes in fullerene chemistry can lead to rather important effects on the mechanical properties of BHJ active layers in OPV. 
Our final example concerns how noncovalent interactions, driven by a combination of the chemistry of $\pi$-conjugated polymer backbones and variations in the chemistry of the alkyl chains appended to the backbone, drive the preferred configurations between fullerenes and copolymers based on alternating electron-rich and electron-poor moieties. A recent study by Graham and co-workers showed that, in the case of the PBDTTPD (composed of alternating thieno[3,4-b]thiophene-pyrrole-4,6-dione and benzodithiophene units) family of polymers, see Figure 4, changes in the nature and positions of the alkyl side-chains affect the efficiency of solar cells with fullerene acceptors to a very significant extent. ${ }^{106}$ As we discussed in the Section 2.4, the locations of the fullerene molecules over the conjugated polymer backbones depend on the nature and position of the alkyl side-chains. ${ }^{162}$ Furthermore, intermolecular interactions between polymer chains and fullerene molecules are known to play crucial roles on the electron-transfer rates, ${ }^{163}$ exciton binding energies, ${ }^{164}$ and processes of charge separation and charge recombination. ${ }^{163}$ Hence, the performance of OPV devices does depend on the intermolecular arrangements and resulting energy landscapes at the interfaces between the polymer and fullerene phases.

As a first step, it was of interest to determine whether, in the absence of any side-chains, a fullerene molecule has a preference to locate over the electron-rich or electron-poor moieties of the copolymer. Thus, we considered a PBDTTPD oligomer without side-chains interacting with $\mathrm{C}_{60}$ in two (face-on and edge-on) orientations, see Figure 12 (all calculations were carried out at the $\omega \mathrm{B} 97 \mathrm{XD} / 6-31 \mathrm{G}(\mathrm{d}, \mathrm{p})$ level of theory, with the coordinates of the oligomer frozen during geometry optimizations of the complexes to limit reconfiguration of the backbone). The difference in interaction energies $(\omega \mathrm{B} 97 \mathrm{XD} / 6-31 \mathrm{G}(\mathrm{d}, \mathrm{p}))$ when $\mathrm{C}_{60}$ sits on top of the electron-rich (BDT) unit with respect to when it sits on top of the electron-poor (TPD) unit is in fact within 
thermal energy at room temperature $(0.4 \mathrm{kcal} / \mathrm{mol}) .{ }^{165}$ Hence, in the absence of alkyl side chains, $\mathrm{C}_{60}$ has no preference on top of the backbone. Edge-on conformations are, however, much less favorable.

\section{$\mathrm{C}_{60}$ on top of electron-rich moiety $\quad-12.3 \mathrm{kcal} / \mathrm{mol}$}

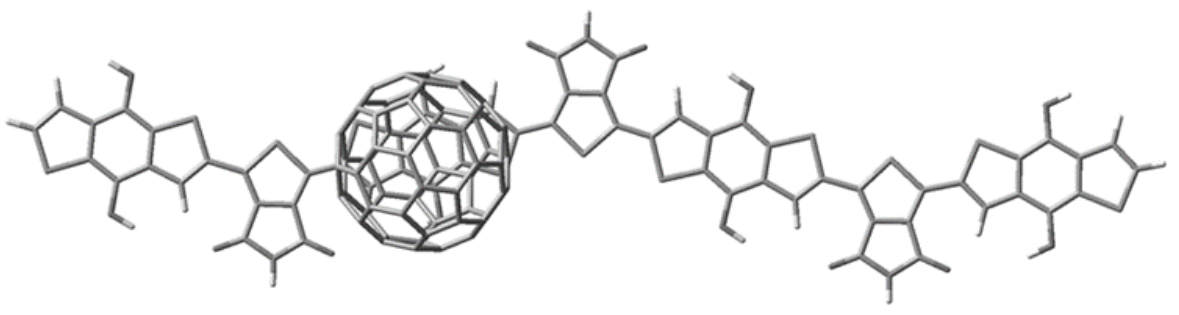

\section{$\mathrm{C}_{60}$ on top of electron-poor moiety $\quad-11.9 \mathrm{kcal} / \mathrm{mol}$}

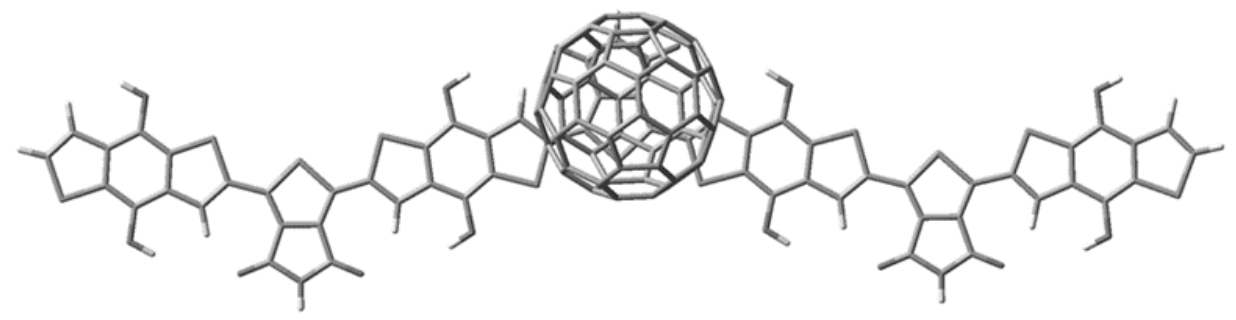

$\mathrm{C}_{60}$ on edge

$-6.3 \mathrm{kcal} / \mathrm{mol}$

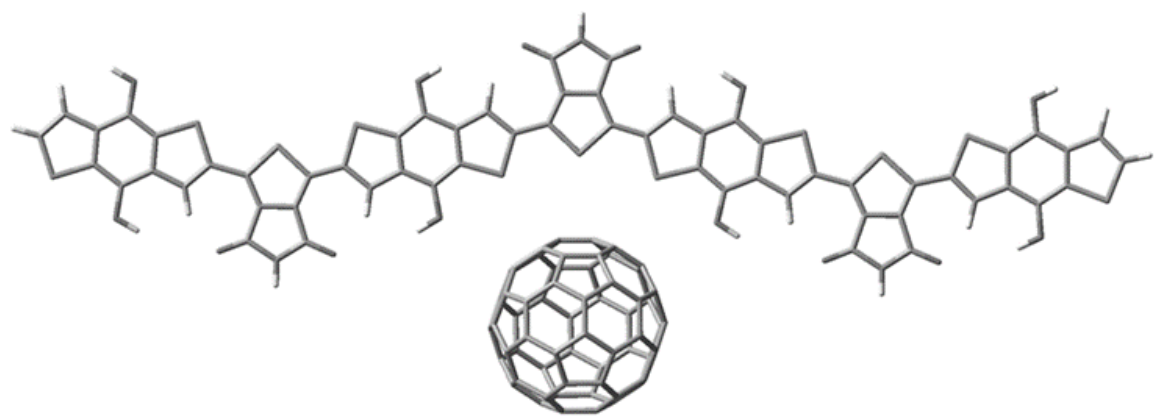

Figure 12. Illustration of the optimized geometries for two face-on configurations and an edgeon configuration of the poly-benzo[1,2-b:4,5-b']dithiophene-thieno[3,4-c]pyrrole-4,6-dione (PBDTTPD) oligomer-fullerene complex at the $\omega \mathrm{B} 97 \mathrm{XD} / 6-31 \mathrm{G}(\mathrm{d}, \mathrm{p})$ level of theory. The calculated counterpoise-corrected interaction energies at $\omega \mathrm{B} 97 \mathrm{XD} / 6-31 \mathrm{G}(\mathrm{d}, \mathrm{p})$ level of theory are also given. ${ }^{165}$ 
Addition of the phenyl butyric acid methyl ester functional group to $\mathrm{C}_{60}$ to form $\mathrm{PC}_{61} \mathrm{BM}$ makes the nature of the interactions between polymer and fullerene more complex. The functional group introduces a dipole moment ${ }^{166,167}$ that adds a significant electrostatic component to the interactions, in addition to the larger dispersion forces. The presence of the attachment of the functional group to the fullerene cage also induces more positional disorder as many orientations of the functional groups are possible, examples of which are shown in Figure $13 .{ }^{168}$

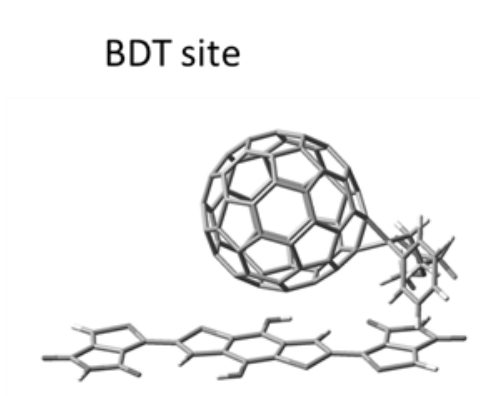

conf-1

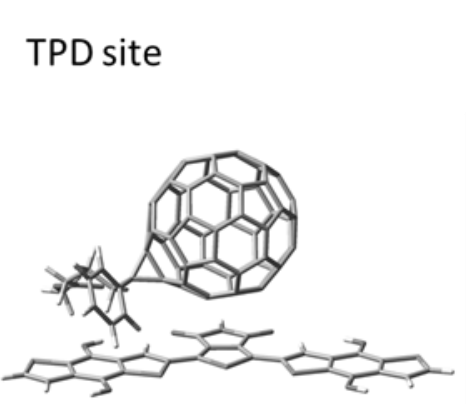

conf-1

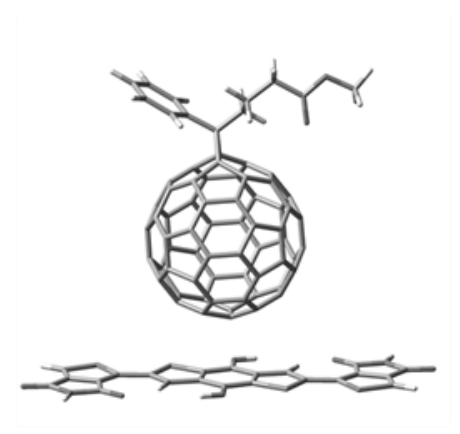

conf-2

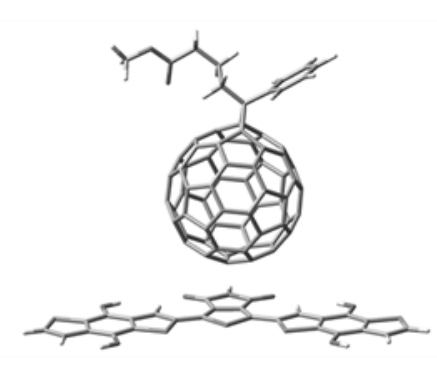

conf-2

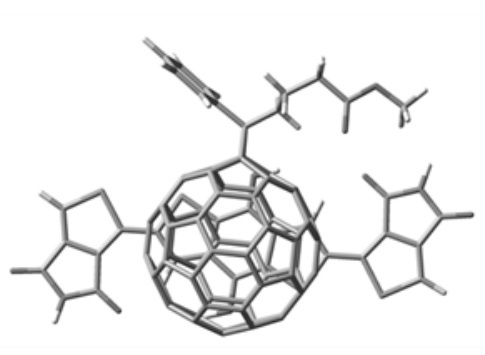

conf-3

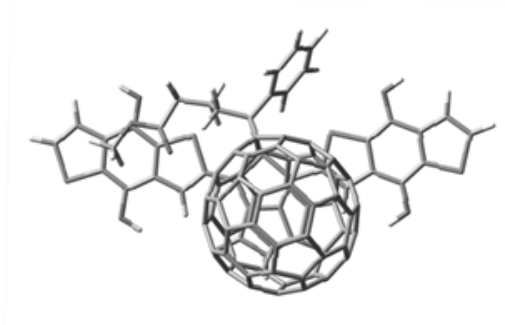

conf-3

Figure 13. Illustration of three typical orientations of $\mathrm{PC}_{61} \mathrm{BM}$ relative to the PBDTTPD backbone, i.e., conf-1, conf-2, and conf-3. (a) and (b) correspond to the cases of $\mathrm{PC}_{61} \mathrm{BM}$ close to either the BDT or TPD moieties of PBDTTPD, respectively. The side-chains of PBDTTPD are not shown for the sake of clarity. ${ }^{168}$ 
MD simulations of the pure phases of the polymers of Figure 4 using the OPLS-AA force field, reveal that linear alkyl side chains on the PBDTTPD backbone tend to extend away from the polymer backbone, while the bulkier branched side-chains remain in closer proximity. ${ }^{168}$ Thus, on average, moieties with linear chains will have more room to accommodate fullerenes than those with branched side-chains. For the blends, the probabilities of finding $\mathrm{PC}_{61} \mathrm{BM}$ close to the BDT and TPD moieties can be extracted from the snapshots of the MD simulations. In going from branched side-chains on the TPD moieties [PBDTTPD $(\mathrm{C} 14-\mathrm{EH})]$ to all linear side-chains [PBDTTPD $(\mathrm{C} 14-\mathrm{C} 8)]$ and then to branched side-chains on the BDT moieties [PBDTTPD(EH$\mathrm{C} 8)$ ], see Figure 4, an increase in the probability of finding $\mathrm{PC}_{61} \mathrm{BM}$ close to the TPD moieties is observed $\left(34.1 \%, 42.3 \%\right.$, and $47.2 \%$, respectively). ${ }^{168}$ The probability of finding $\mathrm{PC}_{61} \mathrm{BM}$ close to the BDT or TPD moiety is mainly determined by interactions between PBDTTPD and $\mathrm{PC}_{61} \mathrm{BM}$, while the orientations of the $\mathrm{PC}_{61} \mathrm{BM}$ on top of the conjugated polymer backbone depend on both PBDTTPD-PC ${ }_{61} \mathrm{BM}$ and $\mathrm{PC}_{61} \mathrm{BM}-\mathrm{PC}_{61} \mathrm{BM}$ interactions.

\section{Synopsis}

We are at the very initial stages of developing the quantum-chemical foundation needed for an $a$ priori computational design of organic semiconductors. The chemistry used to develop organic electronic materials is vast. As highlighted throughout this chapter, seemingly subtle changes in molecular chemical composition and architecture to alter the redox or (opto)electronic properties of isolated molecules can greatly impact the nature and strength of the noncovalent interactions that are important to solubility, solid-state packing, and the macroscopic materials characteristics. We are therefore faced with the significant challenge to develop the multiscale 
models and methods necessary to enable chemical control of these features, in order to improve the design of these important electronically and optically active materials.

\section{Acknowledgements}

This work has been supported in part by King Abdullah University of Science and Technology (KAUST), the KAUST Competitive Research Grant Program, and the Office of Naval Research Global (Award N62909-15-1-2003). We acknowledge the IT Research Computing Team and Supercomputing Laboratory at King Abdullah University of Science \& Technology (KAUST) for providing computational and storage resources. The work at the University of Kentucky was supported by a seed grant from the Center for Applied Energy Research (CAER) and start-up funds provided by the University of Kentucky Vice President for Research. We gratefully thank Drs. Sean Ryno, Naga Rajesh Tummala, and Chris Sutton for stimulating discussions. 


\section{References}

(1) Herwig, P. T.; Müllen, K. Adv. Mater. 1999, 11, 480.

(2) Fournier, D.; Hoogenboom, R.; Schubert, U. S. Chem. Soc. Rev. 2007, 36, 1369.

(3) Hawker, C. J.; Wooley, K. L. Science 2005, 309, 1200.

(4) Cheng, Y.-J.; Yang, S.-H.; Hsu, C.-S. Chem. Rev. 2009, 109, 5868.

(5) Grimsdale, A. C.; Leok Chan, K.; Martin, R. E.; Jokisz, P. G.; Holmes, A. B. Chem. Rev. 2009, 109, 897.

(6) Colson, J. W.; Dichtel, W. R. Nat Chem 2013, 5, 453.

(7) Pope, M.; Kallmann, H. P.; Magnante, P. J. Chem. Phys. 1963, 38, 2042.

(8) Morel, D. L.; Ghosh, A. K.; Feng, T.; Stogryn, E. L.; Purwin, P. E.; Shaw, R. F.; Fishman, C. Appl. Phys. Lett. 1978, 32, 495.

(9) Tang, C. W. Appl. Phys. Lett. 1986, 48, 183.

(10) Tang, C. W.; VanSlyke, S. A. Appl. Phys. Lett. 1987, 51, 913.

(11) Koezuka, H.; Tsumura, A.; Ando, T. Synth. Met. 1987, 18, 699.

(12) Burroughes, J. H.; Bradley, D. D. C.; Brown, A. R.; Marks, R. N.; Mackay, K.; Friend, R. H.; Burns, P. L.; Holmes, A. B. Nature 1990, 347, 539.

(13) Hiramoto, M.; Fujiwara, H.; Yokoyama, M. Appl. Phys. Lett. 1991, 58, 1062.

(14) Garnier, F.; Hajlaoui, R.; Yassar, A.; Srivastava, P. Science 1994, 265, 1684.

(15) Halls, J. J. M.; Walsh, C. A.; Greenham, N. C.; Marseglia, E. A.; Friend, R. H.; Moratti, S. C.; Holmes, A. B. Nature 1995, 376, 498.

(16) Yu, G.; Gao, J.; Hummelen, J. C.; Wudl, F.; Heeger, A. J. Science 1995, 270, 1789.

(17) Friend, R. H.; Gymer, R. W.; Holmes, A. B.; Burroughes, J. H.; Marks, R. N.; Taliani, C.; Bradley, D. D. C.; Santos, D. A. D.; Bredas, J. L.; Logdlund, M.; Salaneck, W. R. Nature 1999, 397, 121.

(18) Horowitz, G. J. Mater. Chem. 1999, 9, 2021.

(19) Hales, J. M.; Matichak, J.; Barlow, S.; Ohira, S.; Yesudas, K.; Brédas, J.-L.; Perry, J. W.; Marder, S. R. Science 2010, 327, 1485.

(20) Gieseking, R. L.; Mukhopadhyay, S.; Risko, C.; Marder, S. R.; Brédas, J.-L. Adv. Mater. 2014, 26,68 .

(21) Gieseking, R. L.; Mukhopadhyay, S.; Shiring, S. B.; Risko, C.; Brédas, J.-L. J. Phys. Chem. C 2014, 118, 23575.

(22) Barlow, S.; Bredas, J.-L.; Getmanenko, Y. A.; Gieseking, R. L.; Hales, J. M.; Kim, H.; Marder, S. R.; Perry, J. W.; Risko, C.; Zhang, Y. Mater. Horiz. 2014, 1, 577.

(23) Arias, A. C.; MacKenzie, J. D.; McCulloch, I.; Rivnay, J.; Salleo, A. Chem. Rev. 2010, 110, 3.

(24) McCulloch, I.; Heeney, M.; Chabinyc, M. L.; DeLongchamp, D.; Kline, R. J.; Cölle, M.; Duffy, W.; Fischer, D.; Gundlach, D.; Hamadani, B.; Hamilton, R.; Richter, L.; Salleo, A.; Shkunov, M.; Sparrowe, D.; Tierney, S.; Zhang, W. Adv. Mater. 2009, 21, 1091.

(25) Diemer, P. J.; Lyle, C. R.; Mei, Y.; Sutton, C.; Payne, M. M.; Anthony, J. E.; Coropceanu, V.; Brédas, J.-L.; Jurchescu, O. D. Adv. Mater. 2013, 25, 6956.

(26) Karamertzanis, P. G.; Day, G. M.; Welch, G. W. A.; Kendrick, J.; Leusen, F. J. J.; Neumann, M. A.; Price, S. L. J. Chem. Phys. 2008, 128, 244708.

(27) Price, S. L. Acc. Chem. Res. 2009, 42, 117.

(28) Virkar, A. A.; Mannsfeld, S.; Bao, Z.; Stingelin, N. Adv. Mater. 2010, 22, 3857.

(29) Wen, S.; Beran, G. J. O. J. Chem. Theory Comput. 2012, 8, 2698.

(30) Li, H.; Tee, B. C. K.; Giri, G.; Chung, J. W.; Lee, S. Y.; Bao, Z. Adv. Mater. 2012, 24, 2588.

(31) Li, H.; Tee, B. C. K.; Cha, J. J.; Cui, Y.; Chung, J. W.; Lee, S. Y.; Bao, Z. J. Am. Chem. Soc. 2012, 134, 2760.

(32) Li, R.; Khan, H. U.; Payne, M. M.; Smilgies, D.-M.; Anthony, J. E.; Amassian, A. Adv. Funct. Mater. 2013, 23, 291. 
(33) Diao, Y.; Tee, B. C. K.; Giri, G.; Xu, J.; Kim, D. H.; Becerril, H. A.; Stoltenberg, R. M.; Lee, T. H.; Xue, G.; Mannsfeld, S. C. B.; Bao, Z. Nat Mater 2013, 12, 665.

(34) Noriega, R.; Rivnay, J.; Vandewal, K.; Koch, F. P. V.; Stingelin, N.; Smith, P.; Toney, M. F.; Salleo, A. Nat Mater 2013, 12, 1038.

(35) Ward, J. W.; Li, R.; Obaid, A.; Payne, M. M.; Smilgies, D.-M.; Anthony, J. E.; Amassian, A.; Jurchescu, O. D. Adv. Funct. Mater. 2014, 24, 5052.

(36) Wei Chou, K.; Ullah Khan, H.; Niazi, M. R.; Yan, B.; Li, R.; Payne, M. M.; Anthony, J. E.; Smilgies, D.-M.; Amassian, A. J. Mater. Chem. C 2014, 2, 5681.

(37) Himmelberger, S.; Duong, D. T.; Northrup, J. E.; Rivnay, J.; Koch, F. P. V.; Beckingham, B. S.; Stingelin, N.; Segalman, R. A.; Mannsfeld, S. C. B.; Salleo, A. Adv. Funct. Mater. 2015, 25, 2616.

(38) Sutton, C.; Risko, C.; Bredas, J.-L. Chem. Mater. 2015, 28, 3.

(39) Takeya, J.; Yamagishi, M.; Tominari, Y.; Hirahara, R.; Nakazawa, Y.; Nishikawa, T.; Kawase, T.; Shimoda, T.; Ogawa, S. Appl. Phys. Lett. 2007, 90, 102120.

(40) Podzorov, V.; Pudalov, V. M.; Gershenson, M. E. Appl. Phys. Lett. 2003, 82, 1739.

(41) Karl, N. Proceedings of the Yamada Conference LVI. The Fourth International Symposium on Crystalline Organic Metals, Superconductors and Ferromagnets (ISCOM 2001). 2003, 133-134, 649.

(42) Le Comber, P. G.; Madan, A.; Spear, W. E. J. Non-Cryst. Solids 1972, 11, 219.

(43) Moore, A. R. Appl. Phys. Lett. 1977, 31, 762.

(44) Paraskar, A. S.; Reddy, A. R.; Patra, A.; Wijsboom, Y. H.; Gidron, O.; Shimon, L. J. W.; Leitus, G.; Bendikov, M. Chem. Eur. J. 2008, 14, 10639.

(45) Stingelin-Stutzmann, N.; Smits, E.; Wondergem, H.; Tanase, C.; Blom, P.; Smith, P.; de Leeuw, D. Nat Mater 2005, 4, 601 .

(46) McGarry, K. A.; Xie, W.; Sutton, C.; Risko, C.; Wu, Y.; Young, V. G.; Bredas, J. L.; Frisbie, C. D.; Douglas, C. J. Chem. Mater. 2013, 25, 2254.

(47) Bergantin, S.; Moret, M. Crystal Growth \& Design 2012, 12, 6035.

(48) Diao, Y.; Shaw, L.; Bao, Z.; Mannsfeld, S. C. B. Energy Environ. Sci. 2014, 7, 2145.

(49) Stone, A. The Theory of Intermolecular Forces; OUP Oxford, 2013.

(50) Hohenstein, E. G.; Duan, J.; Sherrill, C. D. J. Am. Chem. Soc. 2011, 133, 13244.

(51) Ryno, S. M.; Risko, C.; Brédas, J.-L. Chem. Mater. 2016, 28, 3990.

(52) Pauli, W. Zeitschrift für Physik 1925, 31, 765.

(53) Pauli, W. Phys. Rev. 1940, 58, 716.

(54) Wheeler, S. E.; Houk, K. N. Molecular Physics 2009, 107, 749.

(55) Coropceanu, V.; Li, Y.; Yi, Y.; Zhu, L.; Brédas, J.-L. MRS Bulletin 2013, 38, 57.

(56) Coropceanu, V.; Cornil, J.; da Silva Filho, D. A.; Olivier, Y.; Silbey, R.; Brédas, J.-L. Chem. Rev. 2007, 107, 926.

(57) Brédas, J.-L.; Beljonne, D.; Coropceanu, V.; Cornil, J. Chem. Rev. 2004, 104, 4971.

(58) Lee, B.; Chen, Y.; Fu, D.; Yi, H. T.; Czelen, K.; Najafov, H.; Podzorov, V. Nat. Mater. 2013, 12, 1125 .

(59) Liu, Y.; Wang, Y.; Wu, W.; Liu, Y.; Xi, H.; Wang, L.; Qiu, W.; Lu, K.; Du, C.; Yu, G. Adv. Funct. Mater. 2009, 19, 772.

(60) Xiao, K.; Liu, Y.; Qi, T.; Zhang, W.; Wang, F.; Gao, J.; Qiu, W.; Ma, Y.; Cui, G.; Chen, S.; Zhan, X.; Yu, G.; Qin, J.; Hu, W.; Zhu, D. J. Am. Chem. Soc. 2005, 127, 13281.

(61) Liu, Y.; Sun, X.; Di, C.-a.; Liu, Y.; Du, C.; Lu, K.; Ye, S.; Yu, G. Chem. Asian J. 2010, 5, 1550.

(62) Okamoto, T.; Kudoh, K.; Wakamiya, A.; Yamaguchi, S. Chem. Eur. J. 2007, 13, 548.

(63) Tang, M. L.; Okamoto, T.; Bao, Z. J. Am. Chem. Soc. 2006, 128, 16002.

(64) Tang, M. L.; Mannsfeld, S. C. B.; Sun, Y.-S.; Becerril, H. A.; Bao, Z. J. Am. Chem. Soc. 2009, 131,882 .

(65) Laquindanum, J. G.; Katz, H. E.; Lovinger, A. J. J. Am. Chem. Soc. 1998, 120, 664.

(66) Niimi, K.; Shinamura, S.; Osaka, I.; Miyazaki, E.; Takimiya, K. J. Am. Chem. Soc. 2011, 133, 8732. 
(67) Sokolov, A. N.; Atahan-Evrenk, S.; Mondal, R.; Akkerman, H. B.; Sánchez-Carrera, R. S.; Granados-Focil, S.; Schrier, J.; Mannsfeld, S. C. B.; Zoombelt, A. P.; Bao, Z.; Aspuru-Guzik, A. Nat Commun 2011, 2, 437.

(68) Park, J.-I.; Chung, J. W.; Kim, J.-Y.; Lee, J.; Jung, J. Y.; Koo, B.; Lee, B.-L.; Lee, S. W.; Jin, Y. W.; Lee, S. Y. J. Am. Chem. Soc. 2015, 137, 12175.

(69) Okamoto, T.; Kudoh, K.; Wakamiya, A.; Yamaguchi, S. Org. Lett. 2005, 7, 5301.

(70) Yamada, K.; Okamoto, T.; Kudoh, K.; Wakamiya, A.; Yamaguchi, S.; Takeya, J. Appl. Phys. Lett. 2007, 90, 072102.

(71) Pho, T. V.; Yuen, J. D.; Kurzman, J. A.; Smith, B. G.; Miao, M.; Walker, W. T.; Seshadri, R.; Wudl, F. J. Am. Chem. Soc. 2012, 134, 18185.

(72) Mukherjee, A.; Tothadi, S.; Desiraju, G. R. Acc. Chem. Res. 2014, 47, 2514.

(73) Tang, M. L.; Bao, Z. Chem. Mater. 2011, 23, 446.

(74) Mei, J.; Diao, Y.; Appleton, A. L.; Fang, L.; Bao, Z. J. Am. Chem. Soc. 2013, 135, 6724.

(75) Subramanian, S.; Park, S. K.; Parkin, S. R.; Podzorov, V.; Jackson, T. N.; Anthony, J. E. J. Am. Chem. Soc. 2008, 130, 2706.

(76) Battaglia, M. R.; Buckingham, A. D.; Williams, J. H. Chem. Phys. Lett. 1981, 78, 421.

(77) Ringer, A. L.; Sinnokrot, M. O.; Lively, R. P.; Sherrill, C. D. Chem. Eur. J. 2006, 12, 3821.

(78) Holmes, D.; Kumaraswamy, S.; Matzger, A. J.; Vollhardt, K. P. C. Chemistry - A European Journal 1999, 5, 3399.

(79) Sakamoto, Y.; Suzuki, T.; Kobayashi, M.; Gao, Y.; Fukai, Y.; Inoue, Y.; Sato, F.; Tokito, S. J. Am. Chem. Soc. 2004, 126, 8138.

(80) Yagodkin, E.; Xia, Y.; Kalihari, V.; Frisbie, C. D.; Douglas, C. J. J. Phys. Chem. C 2009, 113, 16544.

(81) Moon, H.; Zeis, R.; Borkent, E.-J.; Besnard, C.; Lovinger, A. J.; Siegrist, T.; Kloc, C.; Bao, Z. J. Am. Chem. Soc. 2004, 126, 15322.

(82) Swartz, C. R.; Parkin, S. R.; Bullock, J. E.; Anthony, J. E.; Mayer, A. C.; Malliaras, G. G. Org. Lett. 2005, 7, 3163.

(83) Chikamatsu, M.; Itakura, A.; Yoshida, Y.; Azumi, R.; Yase, K. Chem. Mater. 2008, 20, 7365.

(84) Li, W.; Albrecht, S.; Yang, L.; Roland, S.; Tumbleston, J. R.; McAfee, T.; Yan, L.; Kelly, M. A.; Ade, H.; Neher, D.; You, W. J. Am. Chem. Soc. 2014, 136, 15566.

(85) Fei, Z.; Boufflet, P.; Wood, S.; Wade, J.; Moriarty, J.; Gann, E.; Ratcliff, E. L.; McNeill, C. R.; Sirringhaus, H.; Kim, J.-S.; Heeney, M. J. Am. Chem. Soc. 2015, 137, 6866.

(86) Zhang, X.; Lu, Z.; Ye, L.; Zhan, C.; Hou, J.; Zhang, S.; Jiang, B.; Zhao, Y.; Huang, J.; Zhang, S.; Liu, Y.; Shi, Q.; Liu, Y.; Yao, J. Adv. Mater. 2013, 25, 5791.

(87) Wolf, J.; Cruciani, F.; El Labban, A.; Beaujuge, P. M. Chem. Mater. 2015, 27, 4184.

(88) Jo, J. W.; Jung, J. W.; Wang, H.-W.; Kim, P.; Russell, T. P.; Jo, W. H. Chem. Mater. 2014, 26, 4214.

(89) Carsten, B.; Szarko, J. M.; Son, H. J.; Wang, W.; Lu, L.; He, F.; Rolczynski, B. S.; Lou, S. J.; Chen, L. X.; Yu, L. J. Am. Chem. Soc. 2011, 133, 20468.

(90) Chen, H.-Y.; Hou, J.; Zhang, S.; Liang, Y.; Yang, G.; Yang, Y.; Yu, L.; Wu, Y.; Li, G. Nat Photon 2009, 3, 649.

(91) Stuart, A. C.; Tumbleston, J. R.; Zhou, H.; Li, W.; Liu, S.; Ade, H.; You, W. J. Am. Chem. Soc. 2013, 135, 1806.

(92) Yang, L.; Tumbleston, J. R.; Zhou, H.; Ade, H.; You, W. Energy Environ. Sci. 2013, 6, 316.

(93) Briseno, A. L.; Tseng, R. J.; Ling, M. M.; Falcao, E. H. L.; Yang, Y.; Wudl, F.; Bao, Z. Adv. Mater. 2006, 18, 2320.

(94) Price, S. C.; Stuart, A. C.; Yang, L.; Zhou, H.; You, W. J. Am. Chem. Soc. 2011, 133, 4625.

(95) Schroeder, B. C.; Huang, Z.; Ashraf, R. S.; Smith, J.; D'Angelo, P.; Watkins, S. E.; Anthopoulos, T. D.; Durrant, J. R.; McCulloch, I. Adv. Funct. Mater. 2012, 22, 1663. 
(96) Gundlach, D. J.; Royer, J. E.; Park, S. K.; Subramanian, S.; Jurchescu, O. D.; Hamadani, B. H.; Moad, A. J.; Kline, R. J.; Teague, L. C.; Kirillov, O.; Richter, C. A.; Kushmerick, J. G.; Richter, L. J.; Parkin, S. R.; Jackson, T. N.; Anthony, J. E. Nat Mater 2008, 7, 216.

(97) Do, K.; Saleem, Q.; Ravva, M. K.; Cruciani, F.; Kan, Z.; Wolf, J.; Hansen, M. R.; Beaujuge, P. M.; Brédas, J.-L. Adv. Mater. 2016, Accepted.

(98) Mei, J.; Bao, Z. Chem. Mater. 2014, 26, 604.

(99) Zhang, F.; Hu, Y.; Schuettfort, T.; Di, C.-a.; Gao, X.; McNeill, C. R.; Thomsen, L.; Mannsfeld, S. C. B.; Yuan, W.; Sirringhaus, H.; Zhu, D. J. Am. Chem. Soc. 2013, 135, 2338.

(100) Lei, T.; Dou, J.-H.; Pei, J. Adv. Mater. 2012, 24, 6457.

(101) Anthony, J. E.; Brooks, J. S.; Eaton, D. L.; Parkin, S. R. J. Am. Chem. Soc. 2001, 123, 9482.

(102) Anthony, J. E.; Eaton, D. L.; Parkin, S. R. Org. Lett. 2002, 4, 15.

(103) Sheraw, C. D.; Jackson, T. N.; Eaton, D. L.; Anthony, J. E. Adv. Mater. 2003, 15, 2009.

(104) Ebata, H.; Izawa, T.; Miyazaki, E.; Takimiya, K.; Ikeda, M.; Kuwabara, H.; Yui, T. J. Am. Chem. Soc. 2007, 129, 15732.

(105) El Labban, A.; Warnan, J.; Cabanetos, C.; Ratel, O.; Tassone, C.; Toney, M. F.; Beaujuge, P. M. ACS Appl. Mater. Interfaces 2014, 6, 19477.

(106) Graham, K. R.; Cabanetos, C.; Jahnke, J. P.; Idso, M. N.; El Labban, A.; Ngongang Ndjawa, G. O.; Heumueller, T.; Vandewal, K.; Salleo, A.; Chmelka, B. F.; Amassian, A.; Beaujuge, P. M.; McGehee, M. D. J. Am. Chem. Soc. 2014, 136, 9608.

(107) Dyer-Smith, C.; Howard, I. A.; Cabanetos, C.; El Labban, A.; Beaujuge, P. M.; Laquai, F. Advanced Energy Materials 2015, 5, 1401778/1.

(108) Arnstein, S. A.; Sherrill, C. D. Phys. Chem. Chem. Phys. 2008, 10, 2646.

(109) Sinnokrot, M. O.; Sherrill, C. D. J. Am. Chem. Soc. 2004, 126, 7690.

(110) Sinnokrot, M. O.; Sherrill, C. D. The Journal of Physical Chemistry A 2006, 110, 10656.

(111) Sinnokrot, M. O.; Sherrill, C. D. The Journal of Physical Chemistry A 2004, 108, 10200.

(112) Ringer, A. L.; Figgs, M. S.; Sinnokrot, M. O.; Sherrill, C. D. The Journal of Physical Chemistry A 2006, 110, 10822 .

(113) Marshall, M. S.; Steele, R. P.; Thanthiriwatte, K. S.; Sherrill, C. D. The Journal of Physical Chemistry A 2009, 113, 13628.

(114) Wheeler, S. E.; Houk, K. N. J. Am. Chem. Soc. 2008, 130, 10854.

(115) Wheeler, S. E.; McNeil, A. J.; Müller, P.; Swager, T. M.; Houk, K. N. J. Am. Chem. Soc. 2010, $132,3304$.

(116) Wheeler, S. E. Acc. Chem. Res. 2013, 46, 1029.

(117) Wheeler, S. E.; Bloom, J. W. G. Chemical Communications 2014, 50, 11118.

(118) Grimme, S. Angewandte Chemie International Edition 2008, 47, 3430.

(119) Hohenstein, E. G.; Sherrill, C. D. J. Chem. Phys. 2010, 132, 184111.

(120) Podeszwa, R.; Bukowski, R.; Szalewicz, K. The Journal of Physical Chemistry A 2006, 110, 10345 .

(121) Jeziorski, B.; Moszynski, R.; Szalewicz, K. Chem. Rev. 1994, 94, 1887.

(122) Podeszwa, R.; Szalewicz, K. Phys. Chem. Chem. Phys. 2008, 10, 2735.

(123) Turney, J. M.; Simmonett, A. C.; Parrish, R. M.; Hohenstein, E. G.; Evangelista, F. A.; Fermann, J. T.; Mintz, B. J.; Burns, L. A.; Wilke, J. J.; Abrams, M. L.; Russ, N. J.; Leininger, M. L.; Janssen, C. L.; Seidl, E. T.; Allen, W. D.; Schaefer, H. F.; King, R. A.; Valeev, E. F.; Sherrill, C. D.; Crawford, T. D. WIREs Comput. Mol. Sci. 2012, 2, 556.

(124) The rate at which the interaction energy decreases will be dependent on the separation distance. At separation distances up to 4 angstroms, there will be exponential decrease as charge penetration will be a large contribution. At larger separations, there will be $1 / \mathrm{R}^{\wedge} \mathrm{n}$ dependence due to dispersion and various electrostatic multipole interactions.

(125) The distance dependence of the electrostatics terms will be dependent on the rank of the interacting multipoles. The distance dependence takes the form $1 / \mathrm{R}^{\wedge}(1+\mathrm{L}+\mathrm{M}$ 
(126) Sutton, C.; Marshall, M. S.; Sherrill, C. D.; Risko, C.; Brédas, J.-L. J. Am. Chem. Soc. 2015, 137, 8775 .

(127) Brédas, J. L.; Calbert, J. P.; da Silva Filho, D. A.; Cornil, J. Proceedings of the National Academy of Sciences 2002, 99, 5804.

(128) Sutton, C.; Sears, J. S.; Coropceanu, V.; Brédas, J.-L. J. Phys. Chem. Lett 2013, 4, 919.

(129) Kennedy, M. R.; McDonald, A. R.; DePrince, A. E.; Marshall, M. S.; Podeszwa, R.; Sherrill, C. D. J. Chem. Phys. 2014, 140, 121104.

(130) Anatole von Lilienfeld, O.; Tkatchenko, A. J. Chem. Phys. 2010, 132, 234109.

(131) Wen, S.; Beran, G. J. O. J. Chem. Theory Comput. 2011, 7, 3733.

(132) Podeszwa, R.; Rice, B. M.; Szalewicz, K. Phys. Rev. Lett. 2008, 101, 115503.

(133) Szalewicz, K. Acc. Chem. Res. 2014, 47, 3266.

(134) Huang, L.; Liao, Q.; Shi, Q.; Fu, H.; Ma, J.; Yao, J. J. Mater. Chem. 2010, 20, 159.

(135) Takeshi, M.; Masashi, Y.; Masahito, U.; Masakazu, Y.; Akiko, N.; Yoshinori, T.; Junichi, T.; Yasuo, K.; Yusuke, M.; Takatomo, S. Jpn. J. Appl. Phys. 2010, 49, 085502.

(136) Shigeaki, O.; Toshiaki, M.; Yukihiro, S. Jpn. J. Appl. Phys. 2014, 53, 01 AD02.

(137) A previous computational study predicts three of the four crystal structures found experimentally for rubrene, with a triclinic crystal structure predicted to be the minimum configuration.

(138) In addition to the orthorhombic crystal structure of rubrene, for which large mobilities have been measured, monoclinic and triclinic rubrene polymorphs have been identified. The monoclinic and triclinic polymorphs are not considered in this work for the sake of consistency.

(139) Käfer, D.; Ruppel, L.; Witte, G.; Wöll, C. Phys. Rev. Lett. 2005, 95, 166602.

(140) Petrenko, T.; Krylova, O.; Neese, F.; Sokolowski, M. New J. Phys. 2009, 11, 015001.

(141) Kytka, M.; Gisslen, L.; Gerlach, A.; Heinemeyer, U.; KovaC, J.; Scholz, R.; Schreiber, F. J. Chem. Phys. 2009, 130, 214507.

(142) Casanova, D. J. Chem. Theory Comput. 2014, 10, 324.

(143) Duhm, S.; Xin, Q.; Hosoumi, S.; Fukagawa, H.; Sato, K.; Ueno, N.; Kera, S. Adv. Mater. 2012, 24,901 .

(144) Jurchescu, O. D.; Meetsma, A.; Palstra, T. T. M. Acta Crystallographica Section B 2006, 62, 330.

(145) Norton, J. E.; Brédas, J.-L. J. Am. Chem. Soc. 2008, 130, 12377.

(146) Hallani, R. K.; Thorley, K. J.; Mei, Y.; Parkin, S. R.; Jurchescu, O. D.; Anthony, J. E. Adv. Funct. Mater. 2016, 26, 2341.

(147) Thorley, K. J.; Risko, C. J. Mater. Chem. C 2016, 4, 4040.

(148) Lehnherr, D.; Waterloo, A. R.; Goetz, K. P.; Payne, M. M.; Hampel, F.; Anthony, J. E.; Jurchescu, O. D.; Tykwinski, R. R. Org. Lett. 2012, 14, 3660.

(149) Mamada, M.; Katagiri, H.; Mizukami, M.; Honda, K.; Minamiki, T.; Teraoka, R.; Uemura, T.; Tokito, S. ACS Appl. Mater. Interfaces 2013, 5, 9670.

(150) Mamada, M.; Minamiki, T.; Katagiri, H.; Tokito, S. Org. Lett. 2012, 14, 4062.

(151) Tummala, N. R.; Sutton, C.; Aziz, S. G.; Toney, M. F.; Risko, C.; Bredas, J.-L. Chem. Mater. 2015, 27, 8261.

(152) Hildebrand, J. H.; Scott, R. L. J. Chem. Phys. 1952, 20, 1520.

(153) Hansen, C. M. Journal of Paint Technology 1967, 39, 104.

(154) Tummala, N. R.; Mehraeen, S.; Fu, Y. T.; Risko, C.; Bredas, J. L. Adv. Funct. Mater. 2013, 23,

5800 .

(155) Machui, F.; Abbott, S.; Waller, D.; Koppe, M.; Brabec, C. J. Macromol. Chem. Phys. 2011, 212,

2159.

(156) Machui, F.; Langner, S.; Zhu, X.; Abbott, S.; Brabec, C. J. Sol. Energy Mater. Sol. Cells 2012, 100,138 .

(157) Duong, D. T.; Walker, B.; Lin, J.; Kim, C.; Love, J.; Purushothaman, B.; Anthony, J. E.; Nguyen, T.-Q. Journal of Polymer Science Part B: Polymer Physics 2012, 50, 1405.

(158) Chen, D.; Nakahara, A.; Wei, D.; Nordlund, D.; Russell, T. P. Nano Letters 2010, 11, 561. 
(159) Lin, Y.-H.; Tsai, Y.-T.; Wu, C.-C.; Tsai, C.-H.; Chiang, C.-H.; Hsu, H.-F.; Lee, J.-J.; Cheng, C.Y. Organic Electronics 2012, 13, 2333.

(160) Tummala, N. R.; Bruner, C.; Risko, C.; Brédas, J.-L.; Dauskardt, R. H. ACS Appl. Mater. Interfaces 2015, 7, 9957.

(161) Zhao, Q.; Wood, J. R.; Wagner, H. D. Appl. Phys. Lett. 2001, 78, 1748.

(162) Nielsen, C. B.; Ashraf, R. S.; Treat, N. D.; Schroeder, B. C.; Donaghey, J. E.; White, A. J. P.; Stingelin, N.; McCulloch, I. Adv. Mater. 2015, 27, 948.

(163) Yi, Y.; Coropceanu, V.; Brédas, J.-L. J. Am. Chem. Soc. 2009, 131, 15777.

(164) Linares, M.; Beljonne, D.; Cornil, J.; Lancaster, K.; Brédas, J.-L.; Verlaak, S.; Mityashin, A.; Heremans, P.; Fuchs, A.; Lennartz, C.; Idé, J.; Méreau, R.; Aurel, P.; Ducasse, L.; Castet, F. J. Phys. Chem. C 2010, 114, 3215.

(165) Ravva, M. K.; Wang, T.; Brédas, J.-L. unpublished work 2016.

(166) Marchiori, C. F. N.; Koehler, M. Journal of Physics D: Applied Physics 2014, 47, 215104.

(167) D'Avino, G.; Olivier, Y.; Muccioli, L.; Beljonne, D. J. Mater. Chem. C 2016, 4, 3747.

(168) Wang, T.; Ravva, M. K.; Brédas, J. L. Adv. Funct. Mater. 2016. 